\title{
Turbulent plane wakes subjected to successive strains
}

\author{
By MICHAEL M. ROGERS \\ NASA Ames Research Center, Moffett Field, CA 94035, USA
}

(Received 31 July 2003)

Six direct numerical simulations of turbulent time-evolving strained plane wakes have been examined to investigate the response of a wake to successive irrotational plane strains of opposite sign. The orientation of the applied strain field has been selected so that the flow is the time-developing analogue of a spatially developing wake evolving in the presence of either a favourable or an adverse streamwise pressure gradient. The magnitude of the applied strain rate $a$ is constant in time $t$ until the total strain $e^{a t}$ reaches about four. At this point, a new simulation is begun with the sign of the applied strain being reversed (the original simulation is continued as well). When the total strain is reduced back to its original value of one, yet another simulation is begun with the sign of the strain being reversed again back to its original sign. This process is done for both initially "favourable" and initially "adverse" strains, providing simulations for each of these strain types from three different initial conditions. The evolution of the wake mean velocity deficit and width is found to be very similar for all the "adversely" strained cases, with both measures rapidly achieving exponential growth at the rate associated with the cross-stream expansive strain $e^{a t}$. In the "favourably" strained cases, the wake widths approach a constant and the velocity deficits ultimately decay rapidly as $e^{-2 a t}$. Although all three of these cases do exhibit the same asymptotic exponential behaviour, the time required to achieve this is longer for the cases that have been previously adversely strained (by at $\approx 1$ ). These simulations confirm the generality of the conclusions drawn in Rogers (2002) regarding the response of plane wakes to strain. The evolution of strained wakes is not consistent with the predictions of classical self-similar analysis; a more general 'equilibrium similarity solution' is required to describe the results. At least for the cases considered here, the wake Reynolds number and the ratio of the turbulent kinetic energy to the square of the wake mean velocity deficit are determined nearly entirely by the total strain. For these measures the order in which the strains are applied does not matter and the changes brought about by the strain are nearly reversible. The wake mean velocity deficit and width, on the other hand, differ by about a factor of three when the total strain returns to one, depending on whether the wake was first "favourably" or "adversely" strained. The strain history is important for predicting the evolution of these quantities.

\section{Introduction}

Turbulence models are typically calibrated against turbulent flows in equilibrium or self-similar states. Not surprisingly, they thus often have difficulties in predicting the response of turbulence to "extra" strains such as streamline curvature, streamwise pressure gradients, or cross-flow shear. The presence of competing turbulence production mechanisms results in greater complexity'and predicting the evolution of the turbulence in 
such situations usually requires that additional terms be added to the turbulence model. These terms are often chosen in a somewhat ad hoc manner, as the impact of such extra strains is often poorly understood, providing little guidance to the modeller.

Many experiments have been performed to assess the impact of such extra strains on various canonical turbulent flows. Following Townsend's (1954) study of strained homogeneous turbulence, Reynolds (1962) and $\operatorname{Keffer}(1965,1967)$ used the same distorting wind tunnel to study the impact of global straining on the wakes of various circular cylinders. Other more applied studies for aircraft design have examined the effect of streamwise pressure gradients on wake flows (e.g. Hill, Schaub \& Senoo 1963 and Hoffenberg, Sullivan, \& Schneider 1995). Strained mixing layers have also been studied (Keffer, Kawall, Hunt \& Maxey 1978), as have wakes and mixing layers subjected to cross-plane shear (Nayeri, Beharelle, Delville, Bonnet \& Fiedler 1996, Beharelle, Nayeri, Delville, Bonnet \& Fiedler 1996, and Atsavapranee \& Gharib 1997). In all these experiments the effects of the strain were significant and likely to be challenging for existing turbulence models to predict.

To study the response of free shear flow turbulence to such "extra" strains, Rogers (2002) computed several direct numerical simulations of turbulent plane wakes subjected to global irrotational strains. In these simulations both the orientation of the applied strain and the strain rate were varied. Although the strained wake flows did not evolve according to classical similarity theory, their evolution was found to be consistent with more general 'equilibrium similarity solutions'. The shape of the mean velocity deficit profile was found to be insensitive to the both the orientation and rate of the applied strain. Because of this, the wake Reynolds number was completely determined by the total strain, independent of the strain rate.

In all the strained wake simulations in Rogers (2002), the applied strain rate was constant in time. This constant strain rate is convenient for simplifying the model problem studied because it removes effects associated with a change in strain rate, but it does not correspond to the strain-rate history of practical problems. For instance, in the case of a high-lift multicomponent airfoil the wake of an upstream slat is strained by the pressure field around the main airfoil. The slat wake experiences a short, very strong favorable pressure gradient (expansive streamwise strain) followed by a longer adverse gradient (compressive streamwise strain). Even models that have been developed to account for the effects of extra strains overpredict the slat wake width and velocity deficit (Rumsey \& Gatski 2000).

All the simulations in Rogers (2002) were begun from the same initial unstrained wake flow field. The generality of the conclusions drawn in that work has thus not been demonstrated. Although variations in strain rate relative to the time scale of the initial turbulence were considered, the structure of the initial turbulent field was always the same.

It is thus desirable to address the generality of the results of Rogers (2002) by generating additional simulations, both to study cases with non-constant strain rates and to assess the sensitivity of those results to initial conditions with different turbulent structure. Here results from four additional simulations are studied to address these issues. Rather than selecting an arbitrary strain-rate time history, here the sign of the constant strain rate is simply switched after a certain total strain is reached. A similar flipping of the sign of the strain was employed in an experiment on strained homogeneous turbulence by Gence \& Mathieu (1979), in which the applied strain was generated by passing the flow through a wind tunnel of varying elliptical cross-section. This reversal of the sign of the strain decreases the total strain experienced by the turbulence and this, in turn, 
helps in assessing the relative importance of the strain-rate history on the turbulence as opposed to simply the total strain experienced by the flow.

Gence \& Mathieu (1979) noted that misalignments between the principal axes of the Reynolds stress tensor and the directions of straining last on the order of the inverse strain rate time scale. During the time required for the anisotropy to readjust, flipping the sign of the applied strain causes energy to be transferred from the turbulence back to the mean flow and also drives the Reynolds stresses back towards isotropy. Gence \& Mathieu were able to model the anisotropy evolution quite well using predictions from linear Rapid Distortion Theory (RDT). In RDT the evolution of the Reynolds stresses is governed by the total strain, independent of the strain-rate history. The strained wakes studied here are more complex, with turbulence production by mean wake shear as well as by applied strain. It is thus of interest to determine how the Reynolds stresses respond to flipping the sign of the strain in the sheared inhomogeneous wake turbulence examined here.

In this paper only strains in the plane of the wake mean profile, with no component in the homogeneous spanwise direction, are considered. Such strains are analogous to those experienced by wakes evolving in adverse or favourable streamwise pressure gradients. In Rogers (2002) three "adverse pressure gradient" cases were simulated at various strain rates. To first order, the turbulent statistics were well predicted by the total strain. The mean wake velocity deficit and width exhibited some sensitivity to strain rate, with larger total strains required to achieve the asymptotic similarity growth rate when the strain was applied slowly. Ultimately, though, even weak strains led to exponential variation of the mean wake velocity width and deficit, with the turbulence being unable to evolve rapidly enough to scale with the mean flow. Turbulence measures involving the turbulent kinetic energy, the turbulent kinetic energy dissipation rate, and the turbulent enstrophy exhibited little sensitivity to strain rate, collapsing well as a function of total strain when non-dimensionalized by the instantaneous wake mean velocity deficit and width. This weak dependence on strain rate history suggests that Rapid Distortion Theory (RDT) may be useful for predicting the evolution of strained wakes even for modest strain rates. Indeed Townsend (1980) found that RDT did a good job of predicting the Reynolds stress anisotropy development in the strained wake experiment of Elliott \& Townsend (1981).

By applying the "reversed" strain for the same amount of time as the original strain, the total strain experienced by the turbulence returns to one. At this point the size of the computational domain, which deforms with the mean flow, is the same as that of the original unstrained wake initial flow field but the turbulence, because of irreversible effects associated with the strain history and turbulent diffusion, is different. Thus reversing the sign of the applied strain again at this point will allow a comparison between two flows with the same applied strain rate but different initial conditions. Since two different series of reversing strains are examined in this paper, there are actually three different initial conditions that can be compared.

The additional simulations examined here are thus designed to address several questions. First, to what extent does the order in which various strains are applied matter? Is the final state dependent primarily only on the total strain? Second, to what extent are the effects of such extra strains reversible? If the total strain is returned to one, does the resulting flow resemble that of an unstrained wake that has evolved for the same amount of time? And finally, how universal is the response of different turbulent wakes to the same strain? Are the same asymptotic growth rates of wake width and deficit observed if the initial wake turbulence is different? Does the time required to achieve these asymptotic states vary? 
A brief summary of the governing equations for the time-evolving strained wake, along with possible similarity solutions for this flow, is presented in section 2 . These similarity solutions are derived in greater detail and generality in Rogers (2002). Section 3 contains a brief description of the numerical method used to generate the numerical simulations and a description of the cases studied in this paper. Statistical measures of the turbulence and flow visualizations are examined in section 4 . Conclusions are given in section 5 .

\section{The strained time-evolving wake}

\subsection{The governing equations}

The development of the governing equations for temporally evolving incompressible strained plane wakes is outlined in Rogers (2002). The main results are summarized here for convenience, and to define the notation used in this paper.

The mean velocity field varies in space $x_{i}$ and time $t$ as

$$
\begin{aligned}
& \overline{U_{1}}=a_{11}(t) x_{1}+\bar{U}_{w}\left(x_{2}, t\right) \\
& \overline{U_{2}}=a_{22}(t) x_{2} \\
& \overline{U_{3}}=a_{33}(t) x_{3},
\end{aligned}
$$

where $\overline{U_{i}}$ denotes the $i^{\text {th }}$ component of the mean velocity vector, $\overline{U_{w}}$ is the mean streamwise velocity deficit associated with the wake component of the flow, and $a_{i j}(t)$ indicates the spatially uniform applied mean irrotational strain $\partial \overline{U_{i}} / \partial x_{j}$. The mean continuity equation implies that

$$
\frac{\partial \overline{U_{1}}}{\partial x_{1}}+\frac{\partial \overline{U_{2}}}{\partial x_{2}}+\frac{\partial \overline{U_{3}}}{\partial x_{3}}=a_{11}(t)+a_{22}(t)+a_{33}(t)=0,
$$

thus only two of the irrotational strain components can be selected arbitrarily. The flows examined in this paper have $a_{33}(t)=0$ or, equivalently, $a_{11}(t)=-a_{22}(t)$. Additionally, the strain rates considered here are piecewise constant in time.

For the strained wakes considered here, the mean Navier-Stokes equations reduce to

$$
\frac{\partial \bar{U}_{w}}{\partial t}-a_{22}(t) \bar{U}_{w}+a_{22}(t) x_{2} \frac{\partial \bar{U}_{w}}{\partial x_{2}}+\frac{\partial \overline{u_{1}^{\prime} u_{2}^{\prime}}}{\partial x_{2}}=\nu \frac{\partial^{2} \bar{U}_{w}}{\partial x_{2}^{2}},
$$

with the mean pressure field being described by

$$
\bar{P}=P_{0}-\rho \overline{u_{2}^{\prime 2}}-\frac{\rho}{2} a_{22}^{2}\left(x_{1}^{2}+x_{2}^{2}\right),
$$

(except at the times when the strain is suddenly changed), where $\nu$ is the constant kinematic viscosity, $\rho$ is the constant density, and $a_{33}(t)$ is zero as noted above. The wakes studied here are statistically homogeneous in the streamwise $x_{1}$ and spanwise $x_{3}$ directions, i.e. there are no spatial gradients of turbulent statistics in these coordinate directions. The averages indicated by the overbars are thus taken over $\left(x_{1}, x_{3}\right)$ planes, with primed lower case variables indicating the instantaneous fluctuation about these means. Integrating equation (2.3) from $x_{2}=-\infty$ to $x_{2}=\infty$ yields

$$
A_{w}(t)=A_{w}^{0} \exp \int_{t^{\prime}=0}^{t^{\prime}=t} 2 a_{22}\left(t^{\prime}\right) d t^{\prime}
$$

where

$$
A_{w}(t)=\int_{-\infty}^{\infty} \bar{U}_{w}\left(x_{2}, t\right) d x_{2}
$$


is proportional to the mass flux deficit of the wake.

The governing equation describing the evolution of the Reynolds stresses $\overline{u_{i}^{\prime} u_{j}^{\prime}}$ is given in Rogers (2002). In this paper only the production terms

$$
\begin{aligned}
& \mathcal{P}_{11}=2 a_{22}(t) \overline{u_{1}^{\prime 2}}-2 \overline{u_{1}^{\prime} u_{2}^{\prime}} \frac{\partial \bar{U}_{w}}{\partial x_{2}}=\mathcal{P}_{11}^{s}+\mathcal{P}_{11}^{w} \\
& \mathcal{P}_{22}=-2 a_{22}(t) \overline{u_{2}^{\prime 2}}=\mathcal{P}_{22}^{s} \\
& \mathcal{P}_{33}=0 \\
& \mathcal{P}_{12}=-\overline{u_{2}^{\prime 2}} \frac{\partial \bar{U}_{w}}{\partial x_{2}}=\mathcal{P}_{12}^{w},
\end{aligned}
$$

where the superscripts $s$ and $w$ refer to the strain production and wake shear production terms, respectively, and the dissipation terms

$$
\epsilon_{i j}=-2 \nu \overline{\frac{\partial u_{i}^{\prime}}{\partial x_{k}} \frac{\partial u_{j}^{\prime}}{\partial x_{k}}}
$$

are examined. Twice the turbulent kinetic energy (per unit mass) is defined here as $q^{2}=\overline{u_{i}^{\prime} u_{i}^{\prime}}=\overline{u_{1}^{\prime 2}}+\overline{u_{2}^{\prime 2}}+\overline{u_{3}^{\prime 2}}$ and the dissipation rate of turbulent kinetic energy is denoted by $\epsilon=-\epsilon_{i i} / 2$.

The evolution of a passive scalar quantity with a Prandtl number of $\mathrm{Pr}=0.7$ is calculated along with the development of the hydrodynamic field. For the purposes of this paper, the scalar is used only to assess the level of mixing between the two free streams in flow visualizations (the scalar being zero below the wake and one above it).

\subsection{Self-similarity}

The derivation of both "classical" self-similar solutions and other more general "equilibrium similarity solutions" for temporally evolving strained plane wakes is given in Rogers (2002). The classical similarity solution is derived by assuming that all terms in the various evolution equations are proportional to each other, with the possible exception of the viscous terms, which are often assumed to be negligible. The more general equilibrium similarity solutions are derived by relaxing this assumption and only requiring that certain groups of terms in these equations balance each other. For the classical self-similar solution the wake shear remains proportional to the applied strain rate and the Reynolds stresses all change at the same rate, that rate being proportional to the square of the wake velocity deficit. This is not the case for the equilibrium similarity solutions, in which the wake component of the flow may grow or decay relative to the applied strain rate and the different Reynolds stress tensor components evolve differently. These latter solutions have greater generality than the classical solutions and are found to better describe the strained wake evolution observed in both experiments and numerical simulations (Rogers 2002). A brief summary of these similarity states for constant strain rate and $a_{11}=-a_{22}$ is given in this section. More details for these and other cases, including some with non-constant strain rate $a_{i j}$, can be found in Rogers (2002).

Here the characteristic velocity scale $U_{m}(t)$ is taken to be the maximum value of mean wake velocity deficit and the characteristic length scale $b(t)$ is taken to be the wake halfwidth (here defined as the distance between the $x_{2}$-locations at which the mean velocity deficit is half of $U_{m}$; some investigators take the half-width to be half this distance). For the applied strain considered here with $a_{11}=-a_{22}$, substitution into the classical inviscid self-similar solution for constant strain rate yields

$$
U_{m}(t) \propto e^{a_{22} t}
$$




$$
\begin{aligned}
b(t) & \propto e^{a_{22} t} \\
\eta & =x_{2} / b(t) \\
\bar{U}_{w}\left(x_{2}, t\right) & =-U_{m}(t) f(\eta) \\
\overline{u_{i}^{\prime} u_{j}^{\prime}}\left(x_{2}, t\right) & =e^{2 a_{22} t} k_{i j}(\eta),
\end{aligned}
$$

where $\eta$ is the scaled cross-stream coordinate and $f(\eta)$ and $k_{i j}(\eta)$ are the dimensionless functions characterizing the shapes of the mean velocity deficit and Reynolds stress profiles, respectively. Note that the wake Reynolds number $R e_{m}=U_{m} b / \nu$ grows like $e^{2 a_{22} t}$, as anticipated from equation (2.5).

However, substitution of this solution into equation (2.3) results in the first three terms on the left-hand-side summing to zero. The viscous term is the only term remaining to balance the Reynolds shear stress gradient, but one of these terms is growing while the other is decaying. The classical similarity solution thus does not apply to the strain geometry considered here.

A different equilibrium similarity solution is possible when $U_{m}$ and $b$ scale according to equations (2.9a) and (2.9b). For this solution the peak magnitude of the Reynolds shear stress is constant in time and the time evolution of the normal Reynolds stresses in the scaled $\eta$ coordinate is given by

$$
\begin{aligned}
& \overline{u_{1}^{\prime 2}} \propto e^{2 a_{22} t}\left(1+D_{1}\left(1-e^{-2 a_{22} t}\right)\right) \\
& \overline{u_{2}^{\prime 2}} \propto e^{-2 a_{22} t}\left(1+D_{2}\left(1-e^{2 a_{22} t}\right)\right) \\
& \overline{u_{3}^{\prime 2}} \propto\left(1+D_{3} a_{22} t\right),
\end{aligned}
$$

where $D_{1}, D_{2}$, and $D_{3}$ are dimensionless parameters that may be limited in value to assure realizable Reynolds stresses. The above similarity constraints on the behaviour of $\overline{u_{2}^{\prime 2}}$ and $\overline{u_{3}^{\prime 2}}$ can be eliminated entirely if only the combination (rather than the terms individually) of the pressure strain, dissipation, and turbulent transport terms in the Reynolds stress equation is required to scale like the other terms. However, even then this similarity solution has problems when $a_{22}<0$ because the viscous diffusion terms ultimately grow exponentially relative to the other terms in the equation and cannot be balanced.

Another class of equilibrium similarity solutions can be found by grouping various terms in the evolution equations for the mean momentum and the Reynolds stresses and only requiring that these groups be proportional, rather than requiring each individual term to be proportional. In particular, if the pressure strain, dissipation, and turbulent transport terms in the Reynolds stress equation combine to scale like the wake shear production term and combinations of the strain production term, the advection term, and the time derivative term rather than each scaling like them individually, then possible equilibrium similarity solutions include

$$
\begin{aligned}
U_{m}(t) & \propto e^{a_{22} t} h(t)^{-E_{2}} \\
\frac{b(t)}{u_{1}^{\prime} u_{2}^{\prime}} & \propto e^{a_{22} t} h(t)^{E_{2}} \\
\frac{u_{1}^{\prime 2}}{\frac{u^{2}}{u_{2}^{2}}} & \propto e^{2 a_{22} t} h(t)^{-2 E_{2}} \\
\frac{u_{3}^{\prime 2}}{u^{2}} & \propto h(t)^{-a_{22} t} h(t)^{2 E_{2}-2}
\end{aligned}
$$

where $h(t)=1+E_{1}\left(1-e^{-2 a_{22} t}\right)$ and $E_{1}, E_{2}$, and $E_{3}$ are dimensionless parameters. To 
avoid singularities, when $a_{22}>0$ the value of $E_{1}$ should be at least -1.0 ; when $a_{22}<0$ the value of $E_{1}$ should be zero or negative and physical arguments suggest $E_{2}=\frac{1}{2}$. The results of Rogers (2002) indicate that it is this equilibrium similarity solution that is observed for most strain geometries and confirm that $E_{2}=\frac{1}{2}$ when $a_{22}<0$.

Given that at least the long-time behaviour of the above similarity solutions is exponential, it is convenient to define the dimensionless exponential growth rates

$$
n_{R e}=\frac{1}{R e_{m}} \frac{d R e_{m}}{d(a t)} \quad n_{b}=\frac{1}{b} \frac{d b}{d(a t)} \quad n_{U}=\frac{1}{U_{m}} \frac{d U_{m}}{d(a t)},
$$

where the strain-rate magnitude $a$ is given by $a=\left|a_{11}\right|=\left|a_{22}\right|$. For all the above similarity solutions $n_{R e}= \pm 2$, whereas values for $n_{b}$ and $n_{U}$ differ. For the classical selfsimilar solution and the equilibrium similarity solution (2.10) $n_{b}= \pm 1$ and $n_{U}= \pm 1$, where the plus signs apply when $a_{22}>0$ ("C"-type straining, as defined later) and the minus signs when $a_{22}<0$ ("D"-type straining). For the similarity solution (2.11) $n_{b}=1$ and $n_{U}=1$ when $a_{22}>0$ and $n_{b}=2 E_{2}-1$ and $n_{U}=-2 E_{2}-1$ when $a_{22}<0$ (the value $E_{2}=\frac{1}{2}$ yielding $n_{b}=0$ and $n_{U}=-2$ ).

\section{Generating the simulations}

\subsection{The numerical method}

In the absence of an applied strain, both the streamwise and spanwise directions of a temporally evolving wake are homogeneous, with the wake spreading in the inhomogeneous cross-stream direction as time evolves. However, application of a global mean strain generates additional terms in the governing equations that exhibit a direct dependence .on the spatial coordinates $x_{i}$ and therefore prevent homogeneity. As shown by Rogallo $(1977,1981)$, this explicit dependence can be removed by transforming to a moving coordinate system that follows the mean velocity field associated with the applied strain. In this moving reference frame the problem again becomes homogeneous in the mapped streamwise and spanwise directions and Fourier expansions can be used to represent the spatial variation of the dependent variables in these directions.

In this moving coordinate system the numerical method used to simulate unstrained wakes in Moser et al. (1998) becomes computationally unfeasible. Instead, the crossstream dependence of the flow variables is handled by the method developed by Corral \& Jimenez (1995). For this method, the computational domain is chosen to be large enough so that the vorticity is confined to a region away from the cross-stream boundaries. Periodicity is assumed in the cross-stream direction for the purposes of time-advancing the vorticity field, but the effect of the undesired periodic image flows is removed by using an appropriately corrected velocity field when computing the nonlinear terms. The mathematical details of combining the transformation developed by Rogallo $(1977,1981)$ with the method of Corral \& Jimenez (1995) to simulate strained inhomogeneous free shear flows are outlined in an appendix of Rogers (2002), available from the JFM Editorial Office at Cambridge.

The number of Fourier modes required to adequately resolve the turbulence in each simulation is variable, depending on the orientation and duration of the strain. Because the computational domain is deforming with the mean strain, it eventually becomes necessary to add computational modes in directions of stretching and it may be possible to remove modes in directions of compression or as the flow structures increase in scale. Such "remeshing" is done several times during each simulation to ensure efficient use of the computational resources (between 4 and 14 times for each of the six cases). The number of Fourier modes at the start and finish of each calculation is given in table 1. To 


\begin{tabular}{|c|c|c|c|c|c|c|c|c|c|c|c|}
\hline case & $\mid N_{x_{1}}^{i}$ & $\Lambda$ & & $N_{\mathbf{x}_{1}}^{f}$ & $N_{x_{2}}^{f}$ & $N_{x_{3}}^{f} \mid$ & $\tau_{\boldsymbol{i}}$ & $\tau_{f}$ & $|a \Delta t|$ & $\mid e^{a \Delta}$ & \\
\hline & 512 & 240 & 128 & & 700 & 64 & 3.16 & $\mid 10.17$ & $\mid 1.90$ & 6.7 & 107 \\
\hline Case D & 512 & 240 & 128 & 1536 & 128 & 64 & 3.16 & 11.64 & 2.30 & 10.0 & 72 \\
\hline Case CD & 96 & 600 & 96 & 1920 & 128 & 80 & 8.32 & 23.82 & 4.20 & 66.7 & 163 \\
\hline Case DC & 768 & 144 & 80 & 384 & 400 & 64 & 8.32 & 14.96 & 1.80 & 6.0 & 37 \\
\hline Case CDC & 256 & 320 & 96 & 128 & 512 & 96 & 13.49 & 18.65 & 1.40 & 4. & 50 \\
\hline Case DCD & 512 & 400 & 64 & 1920 & 128 & 64 & 13.49 & 31.20 & 4.80 & 121.5 & 81 \\
\hline
\end{tabular}

TABLE 1. Number of modes in initial mesh $\left(N^{i}\right)$, after final remesh $\left(N^{f}\right)$, initial $\left(\tau_{i}\right)$ and final $\left(\tau_{f}\right)$ times of the time interval over which the strain is applied, total strain achieved by each case $\left(\Delta t=t_{f}-t_{i}\right)$, and Cray C90 CPU hour requirement for each simulation. The strain geometry for cases ending in " $\mathrm{C}$ " is compressive in $x_{1}$ and expansive in $x_{2}$; this is reversed for cases ending in " $\mathrm{D}$ ". The strain rate magnitude for all cases is $a b^{0} / U_{m}^{0}=0.271$.

fully dealias the results, the "physical space" grids used to compute nonlinear products contain 3/2 as many grid points as the number of Fourier modes listed for each of the coordinate directions. The number of Cray C90 CPU hours required for each of the different cases is also given in table 1.

\subsection{Cases simulated}

The strained wake cases simulated here all result from the application of spatially uniform and temporally piecewise constant strains in the $x_{1}-x_{2}$ plane. The initial unstrained wake flow field to which strain is first applied is the same as the one used in Rogers (2002). The reasoning for selecting this particular initial field is given there. Basically, the field with the largest statistical sample of eddies (or, equivalently, the smallest wake width for a given computational domain size) that is still in the self-similar evolution period of the unstrained wake calculation was chosen (see Moser et al. 1998).

At time $t_{1}$, the first constant strain is applied. The two cases examined here are cases C and $D$ of Rogers (2002), and are referred to here by the same name. As noted above, for these flows the applied strain is in the $x_{1}-x_{2}$ plane, with no component in the spanwise direction. The strain in case $\mathrm{C}$ is compressive in the streamwise direction and expansive in the cross-stream direction, corresponding to the strain felt by a spatially developing wake entering a region of adverse pressure gradient (for this reason " $\mathrm{C}$ "-type straining will also be referred to as "adverse" straining). The streamwise computational domain size $L_{x}$ thus decreases with time (or increasing total strain) and eventually artificially constrains the flow evolution compared to the infinite-domain problem being modeled. This computational limitation is further compounded by the rapidly increasing wake width associated with the expansive cross-stream strain. By $a\left(t-t_{1}\right) \approx 1.0$, or a total strain of 2.7 , the width of vortical fluid is comparable to $L_{x}$ and beyond this time large-scale eddies (of scale $L_{x}$ ) will be impacted by the periodic boundary conditions of the simulations. However, examination of the vortical structures prior to this point in the evolution suggests that such large-scale motions do not form for this orientation of the applied strain. Apparently the wake is pulled out too rapidly in the cross-stream direction for coherent, slow, large-scale structures of this scale to form. Because of this, the simulations are probably not strongly impacted by the limited streamwise domain until later and the run was not discontinued until $a\left(t-t_{1}\right)=1.9$.

In case $\mathrm{D}$ the strain has the opposite sign (but same magnitude and orientation), with 
expansive strain in the streamwise direction and compressive strain in the cross-stream direction. This is analogous to the straining caused by a favourable pressure gradient and " $\mathrm{D}$ "-type straining is also referred to here as "favourable" straining. In this case, the streamwise computational domain extent $L_{x}$ increases and the statistical sample of eddies thus also increases throughout the run (although not as rapidly as the box size since the eddies become elongated in the streamwise direction). Despite the compressive strain in $x_{2}$, the wake width does not become negligible and this computation could be continued indefinitely. However, because of increasing computational cost associated with the growing domain size, the simulation was terminated at $a\left(t-t_{1}\right)=2.3$.

The evolutions of cases $C$ and D have been documented in Rogers (2002). The new simulations considered in this paper consist of four new cases in which the strain is suddenly changed to a new value at a time $t_{2}$ (cases CD and DC) or $t_{3}$ (cases CDC and DCD). Given the limitations on case $\mathrm{C}$ described above, $t_{2}$ was chosen such that $a\left(t_{2}-t_{1}\right)=1.4$, corresponding to a total strain of about 4 . In order to look for effects of the strain history, it is desirable to have this total strain be large, but if it were any larger than this it would be hard to separate differences resulting from the strain history from those associated with the finite computational domain.

The initial condition for case CD is the flow of case C at $t_{2}$. At this time the "adverse" straining is switched to "favourable" straining of the same magnitude and the computation is continued. Note that case $C$ is also continued further until $a\left(t-t_{2}\right)=0.5$, as noted above. Similarly, the initial condition for case DC is the flow of case D at $t_{2}$, at which time the "favourable" straining of case D is switched to "adverse" straining of the same magnitude. Case $\mathrm{D}$ is further continued until $a\left(t-t_{2}\right)=0.9$.

At time $t_{3}$, chosen such that $t_{3}-t_{2}=t_{2}-t_{1}=\Delta t$, the total strain experienced by both cases $\mathrm{CD}$ and $\mathrm{DC}$ is one. The computational domain in fixed laboratory coordinates is the same size as that of the unstrained wake simulation used as an initial condition at time $t_{1}$. It is instructive to compare cases $\mathrm{CD}$ and $\mathrm{DC}$ at this time. If the wake turbulence is affected primarily by the total strain, then these two flows should be similar at $t_{3}$. If strain history has a significant impact on the evolution then there should be notable differences.

At time $t_{3}$, the reversal of the sign of the strain is repeated. The "favourable" strain of case CD is switched to "adverse" for case CDC and the "adverse" strain of case DC is switched to "favourable" for case DCD. Again, the magnitude of the strain rate $a$ remains unchanged. Case CD is continued until $a\left(t-t_{3}\right)=2.8$ and case DC is continued until $a\left(t-t_{3}\right)=0.4$. For the reasons discussed above, computational domain size limitations necessitate termination of case DC relatively quickly, whereas case $C D$ could be run indefinitely. The new cases CDC and DCD are run until $a\left(t-t_{3}\right)=1.4$ and $a\left(t-t_{3}\right)=4.8$, respectively. Case CDC is thus terminated at $t_{4}=t_{3}+\Delta t$, whereas case DCD is run until $a\left(t-t_{4}\right)=3.4$.

Comparison of case CDC with case $\mathrm{C}$ and of case $\mathrm{DCD}$ with case $\mathrm{D}$ is also instructive. All four of these simulations begin from a total strain of one, but the initial turbulence is different between the previously unstrained and the successively strained flows. For cases $\mathrm{C}$ and $\mathrm{D}$ the initial turbulence is that associated with a self-similar unstrained wake, whereas for cases CDC and DCD the initial turbulence has undergone fairly rapid squeezing and stretching (resulting in thicker wakes with smaller mean velocity deficits).

The strain rate for all the computations is chosen to be the same as that used in the previous strained wake simulations of Rogers (2002), namely $a b^{0} / U_{m}^{0}=0.271$, where $a$ corresponds to the magnitude of the strain rate in both of the two strained coordinate directions, and $b^{0}$ and $U_{m}^{0}$ are the wake width and velocity deficit, respectively, at the time $t_{1}$ when the first strain is initially applied. This choice of $a$ is roughly equal to the 


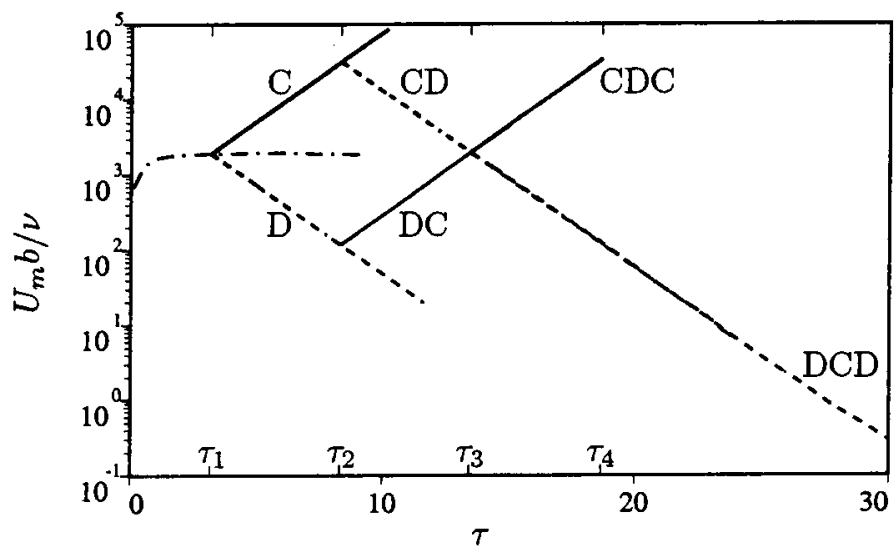

Figure 1. Evolution of the wake Reynolds number $R e_{m}=U_{m} b / \nu .-$ "C" strain, -... "D" strain, - - - no strain.

initial inverse turbulence time scale $\epsilon / q^{2}$, with the initial value of the dimensionless ratio $a q^{2} / \epsilon$ being 1.06 at the centreline when $t=t_{1}$. The quantities $b^{0}$ and $U_{m}^{0}$ are also used to define a dimensionless time $\tau=t U_{m}^{0} / b^{0}$. The dimensionless times $\tau$ at which each case is begun and terminated, along with the total strain achieved during that time interval are listed in table 1.

\section{Results from the simulations}

In this section both turbulence statistics and contour plots of the spanwise vorticity component are examined to study the impact of successively applied strains on the wake. The generality of the behaviour observed in Rogers (2002) and the sensitivity of various statistics to changes in the initial conditions are assessed.

\subsection{The wake mean velocity deficit}

As noted in section 2.2, the wake Reynolds number $R e_{m}$ based on $b$ and $U_{m}$ should grow exponentially if the shape of the mean wake velocity profile is universal. The evolution of the Reynolds number $R e_{m}$ is shown in figure 1 on a log-linear scale to facilitate the determination of exponential behaviour. The time coordinate has been non-dimensionalized as $\tau=t U_{m}^{0} / b^{0}$, as noted previously (note $\tau_{1}=t_{1} U_{m}^{0} / b^{0}=3.16$ ). The Reynolds number of the unstrained wake (chain-dotted line) rapidly approaches a constant value, as expected for a self-similar wake, of about 2000 . The strain is applied at the beginning of what is deemed to be the "self-similar period" of this unstrained simulation, within the period of nearly constant Reynolds number. The three flows with "C"-type strain $\left(a_{22}>0\right)$ are shown by solid lines and those with " $D$ "-type strain $\left(a_{22}<0\right)$ are shown by dashed lines. All the curves for the strained cases are linear on this log-linear plot, confirming that all the cases exhibit exponential growth. This exponential growth begins immediately upon application of the strain, without any transitional period. Also, as predicted by the self-similar analysis for strained wakes, the growth rate for the "C"- and "D"-type cases is $n_{R e}=2$ and $n_{R e}=-2$, respectively.

The agreement between the analytical self-similar solution and the results presented in figure 1 suggests that the mean velocity profile shape is universal, as assumed in equation $(2.9 \mathrm{~d})$. This is confirmed in figure 2 , in which the scaled mean velocity profiles are shown for each flow at various times. The mean velocity profile shapes are similar, both over time as each flow develops and between the different cases. These shapes are 

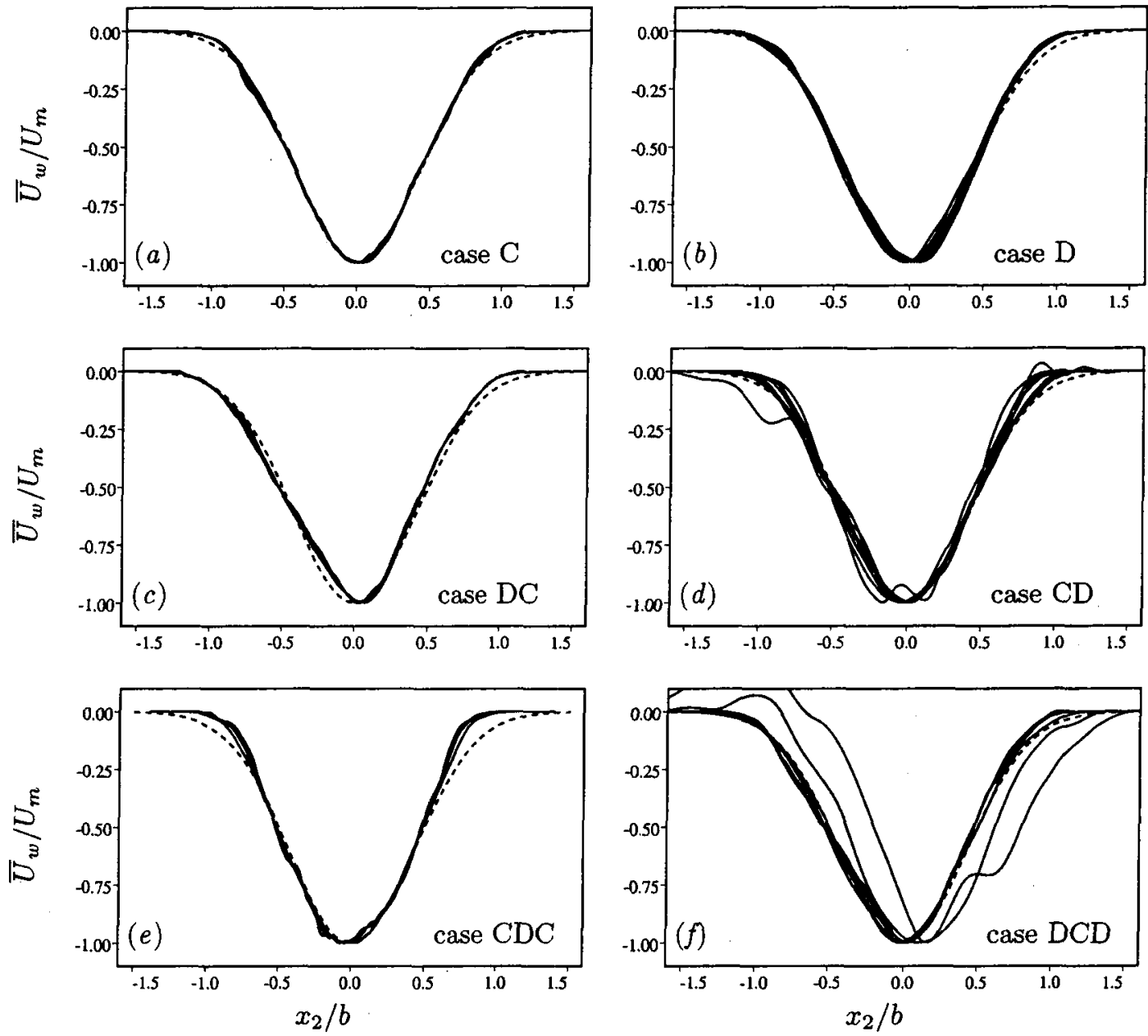

FIGURE 2. Scaled wake mean velocity profiles at several roughly equally spaced times during each simulation for $(a)$ case $\mathrm{C},(b)$ case $\mathrm{D},(c)$ case $\mathrm{DC},(d)$ case $\mathrm{CD},(e)$ case $\mathrm{CDC}$, and $(f)$ case DCD. The ---- curve indicates a gaussian.

close to gaussian, but with slightly more rapid decay at the edges of the wake, as is also observed for unstrained wakes. Exceptions at late times in cases CD and DCD are a result of noise; the peak level of the velocity deficit, $U_{m}$, has dropped by three orders of magnitude for these cases (since the strain was first applied at $\tau_{1}$ ) and the wake is essentially gone. Note that $R e_{m}$ is not sensitive to these late time oscillations in the mean velocity profile.

The shape of the mean velocity profile remains unchanged when each strain is first applied, throughout the straining, and when a different strain is imposed. Because of this, there is no transitional period required to achieve the self-similar growth rate $n_{R e}= \pm 2$. Thus the strain history is not important for determining the further evolution of $R e_{m}$. The order in which the different strains are applied is seen to be irrelevant in figure 1 , with $R e_{m}$ being uniquely determined by the total strain at any given time $\tau$. For both cases CD and DC, reversing the sign of the strain at $\tau_{2}=8.32$ for another $a \Delta t$ of 1.4 to $\tau_{3}=13.49$ leads to the same value of $R e_{m}$ as observed at $\tau_{1}$. Also, the $R e_{m}$ evolutions 


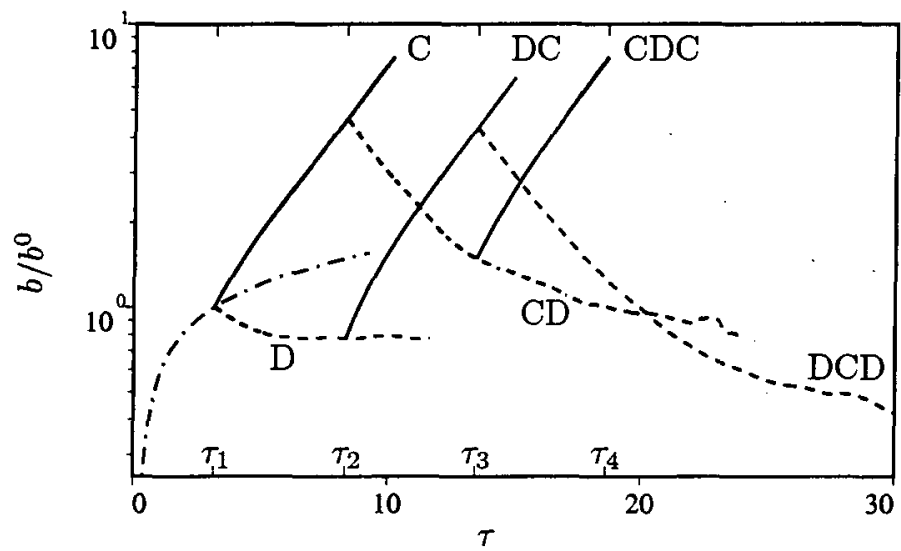

FIguRE 3. Evolution of the wake width $b$, normalized by $b^{0}$, its value at $\tau_{1} . \longrightarrow$ " $\mathrm{C}$ " strain, --- "D" strain, - - no strain.

for the continuation of these two simulations beyond this time are identical to those of cases CDC and DCD.

The time evolution of the wake width $b$ is shown in figure 3 , again plotted in loglinear coordinates and again with the unstrained wake results included for comparison. After a transitional period, the widths in the "C"-type cases grow exponentially with $n_{b}=1$. The transition time required to achieve this state of exponential growth is about the same for all three cases. The widths in the " $D$ "-type cases initially decay and then approach a constant. In cases $C D$ and $D C D$ there is a fairly extended period ( $a \Delta t \approx 1)$ of exponential decay with $n_{b}=-1$ (the decay rate predicted by classical similarity theory) before $n_{b}$ approaches zero. The widths in these two cases do not exhibit an extended period with $n_{b}=0$, although at these late times the wake is essentially gone, with the deficit being less than $1 \%$ of its initial amplitude at $\tau_{1}$. The asymptotic state does appear to be characterized by $n_{b}=0$, but the transition time required to achieve this and the period of $n_{b}=-1$ behaviour are different from the behaviour observed in case D.

Because the response in case $\mathrm{D}$ is somewhat different from that observed in cases $\mathrm{CD}$ and $\mathrm{DCD}$, the ultimate state of wakes undergoing " $\mathrm{D}$ "-type straining may be sensitive to the initial conditions and the strain history, rather than just to the total strain. For instance, the wake width at $\tau_{3}$ for case DC is greater than that at the same time for case $C D$, despite the total strain being one in both cases. In fact, the width at $\tau_{3}$ for case DC is $93 \%$ of that of case $\mathrm{C}$ at $\tau_{2}$, even though the latter flow has not been compressed in the cross-stream direction at any time during its evolution. Extrapolating the unstrained wake evolution (chain-dotted curve) in figure 3 suggests a wake width somewhere between those of cases CD and DC at $\tau_{3}$. Thus the order in which the wake is "adversely" or "favourably" strained has a significant impact on the wake width; in this case resulting in wake widths that are about a factor of three different at $\tau_{3}$.

Similar conclusions hold for the peak wake velocity deficit $U_{m}$, shown in figure 4 . After a brief transitional period, the deficits in the " $\mathrm{C}$ "-type cases grow exponentially with $n_{U}=1$, whereas those in the " $\mathrm{D}$ "-type cases ultimately decay at a rate approaching $n_{U}=-2$, with cases $\mathrm{CD}$ and $\mathrm{DCD}$ again exhibiting a longer transient period (with $n_{U} \approx-1$ ). As with the wake width, this results in values of the wake deficit that are dependent on the strain history, and not just on the total strain (again about a factor of three difference between cases CD and DC at $\tau_{3}$ ).

The wake shear rate, characterized by the ratio $U_{m} / b$, is plotted in figure 5 , nondimensionalized by the constant strain rate $a$. The shear rates for all three "C"-type 


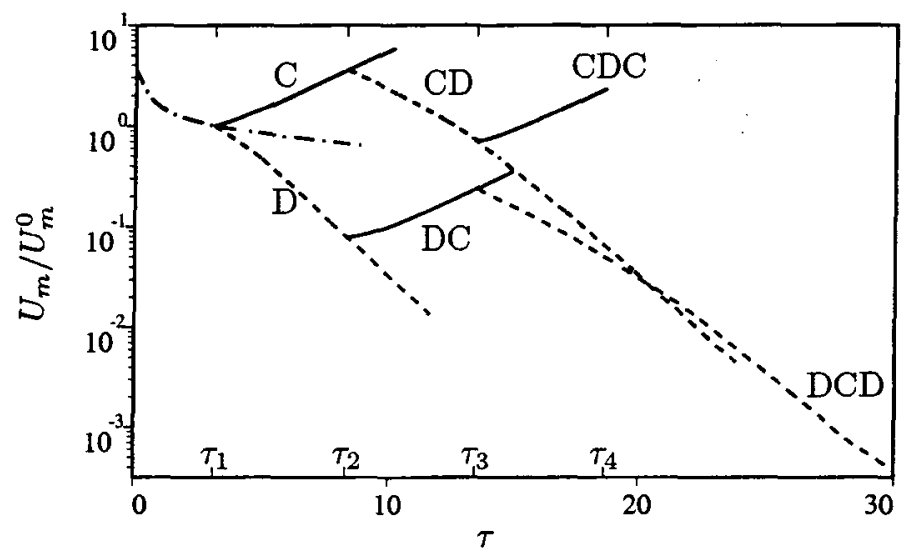

FIGURE 4. Evolution of the peak wake velocity deficit $U_{m}$, normalized by $U_{m}^{0}$, its value at $\tau_{1}$. "C" strain, - - - "D" strain, - - no strain.

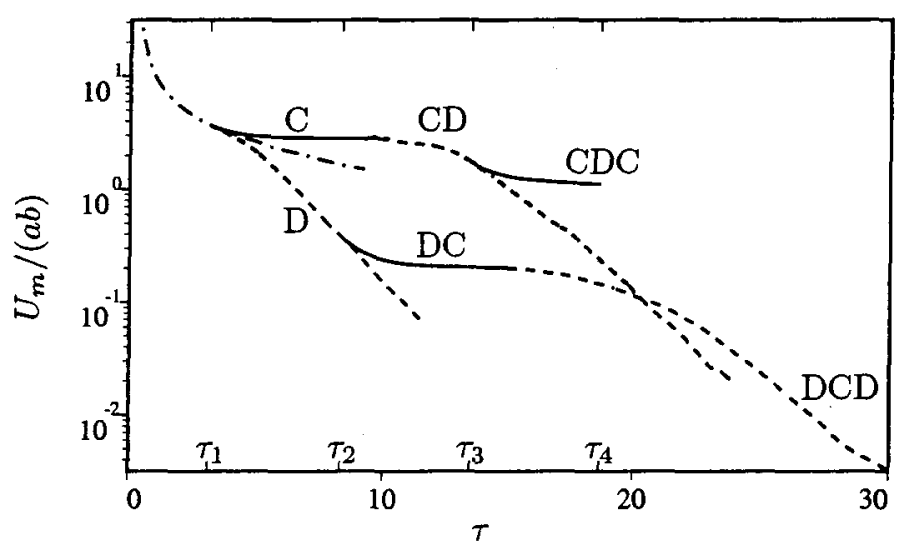

FIGURE 5. Ratio of wake shear rate to applied strain rate $U_{m} /(a b)$. - $C$ " strain, - - - "D" strain, - - - no strain.

cases ultimately approach a constant. In these three flows the wake shear rate comes into balance with the constant applied strain rate, as predicted by all of the similarity solutions presented in section 2.2. The cases with "D"-type strain all exhibit rapidly decaying wake shear rates, indicating that the wakes in these flows are strongly suppressed by the applied strain. The wake component of these flows becomes insignificant and the evolutions are essentially those of a strained slab of turbulence. The strain-rate history is important in determining the level of the shear-strain ratio. At $\tau_{3}$ (total strain of one), $U_{m} /(a b)=0.203$ for case DC, only $12 \%$ that of case CD (1.735) at the same time and total strain. A fixed amount of total "favourable" strain is thus apparently more efficient at suppressing a wake if applied prior to any "adverse" straining. As can be seen in figure 5, the decay in the shear rate is delayed for cases CD and DCD (compared to that of case D) after the application of "favourable" strain at $\tau_{2}$ and $\tau_{3}$, respectively.

\subsection{The turbulence levels}

According to the classical self-similar solution of section 2.2, the turbulent kinetic energy should scale with the square of the wake mean velocity deficit. The evolution of the maximum value of $q^{2}$ in $x_{2}$ (denoted by $q_{m}^{2}$ ) scaled in this way is shown in figure $6 \mathrm{a}$. As expected, the ratio is roughly constant for the developed unstrained wake (chain-dotted 

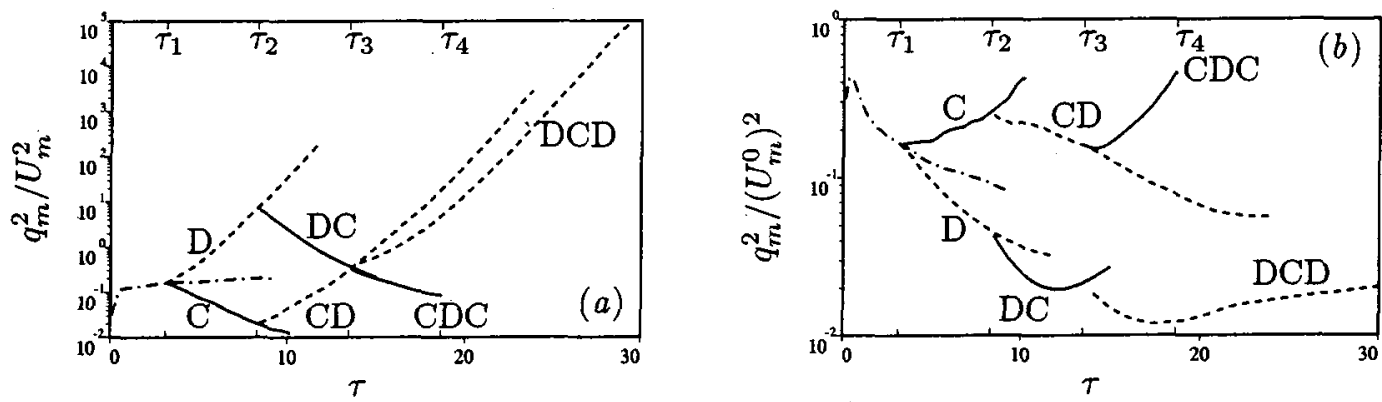

FIGURE 6. Turbulence kinetic energy evolution at point of maximum $q^{2}$, (a) scaled by the wake deficit $U_{m}$ and $(b)$ normalized by the value of the wake deficit at $\tau_{1}, U_{m}^{0}$. - - - "D" strain, _- - no strain.

curve). On the other hand, the ratio is not constant for any of the strained flows. As noted in Rogers (2002), the turbulence levels cannot keep pace with the rapidly changing mean wake velocity deficit. For the "C"-type cases, in which the deficit increases, the ratio $q_{m}^{2} / U_{m}^{2}$ decays, while for the " $\mathrm{D}$ "-type cases, in which the deficit decays, the ratio increases.

The evolution of the unscaled value of $q_{m}^{2}$ (non-dimensionalized by $\left(U_{m}^{0}\right)^{2}$, the square of the velocity deficit at $\tau_{1}$ ) is shown in figure $6 \mathrm{~b}$. Consistent with the above discussion, the variation in $q_{m}^{2} /\left(U_{m}^{0}\right)^{2}$ is much less than that of $U_{m}^{2}$, with the data from all cases lying between 0.01 and 0.5 , instead of spanning more than four orders of magnitude. For the " $\mathrm{C}$ "-type cases, the peak turbulent kinetic energy levels ultimately increase (faster than exponentially over the periods computed), whereas for the " $D$ "-type cases these levels initially decrease, perhaps becoming roughly constant at late times (or even slowly increasing for case $\mathrm{DCD}$ ). Even for cases $\mathrm{C}$ and $\mathrm{CDC}$, the kinetic energy levels increase by only a factor of three throughout the entire simulation (although these increased energy levels are maintained across wakes of rapidly increasing width).

It is remarkable that although the levels of $q_{m}^{2} /\left(U_{m}^{0}\right)^{2}$ for cases CD and DC are nearly an order of magnitude different at $\tau_{3}$, the corresponding values of $q_{m}^{2} / U_{m}^{2}$ are virtually identical. After $\tau_{3}$, the evolution of $q_{m}^{2} / U_{m}^{2}$ for case DC closely matches that of case CDC. The agreement for cases CD and DCD is less impressive but still notable, especially considering that the wake has been largely suppressed for these cases. For these strained wakes the level of $q_{m}^{2} / U_{m}^{2}$ is thus reasonably well predicted by the value of the total strain. The evolution of the turbulence kinetic energy level can thus be calculated if the evolution of the mean velocity deficit can be determined.

The evolution of the maximum (in $x_{2}$ ) turbulent kinetic energy dissipation rate, $\epsilon_{m}$, is qualitatively similar to that of the turbulent kinetic energy $q_{m}^{2}$. In cases D and CD, $\epsilon_{m}$ decays rapidly; in case DCD initial decay is followed by a slow increase. The dissipation rate $\epsilon_{m}$ ultimately increases for the "C"-type cases, although this follows a significant period of decay, particularly for case DC. Scaling $\epsilon_{m}$ by $U_{m}^{3} / b$ greatly reduces the disparity between the different cases with the same total strain, but $\epsilon_{m} b / U_{m}^{3}$ is not as convincingly dependent on only the total strain as $q_{m}^{2} / U_{m}^{2}$; there is some dependence on the strain history.

The rough similarity in the growth behaviour of the kinetic energy and its dissipation rate results in a time scale $q^{2} / \epsilon$ that changes relatively slowly compared to the other turbulence statistics examined here. The value of the time scale at the point of maximum (in $x_{2}$ ) turbulent kinetic energy, $q_{m}^{2} / \epsilon_{q}$, is shown in figure $7(a)$, scaled by the constant strain-rate magnitude $a$. Note that this figure is plotted on a linear scale, and that the 

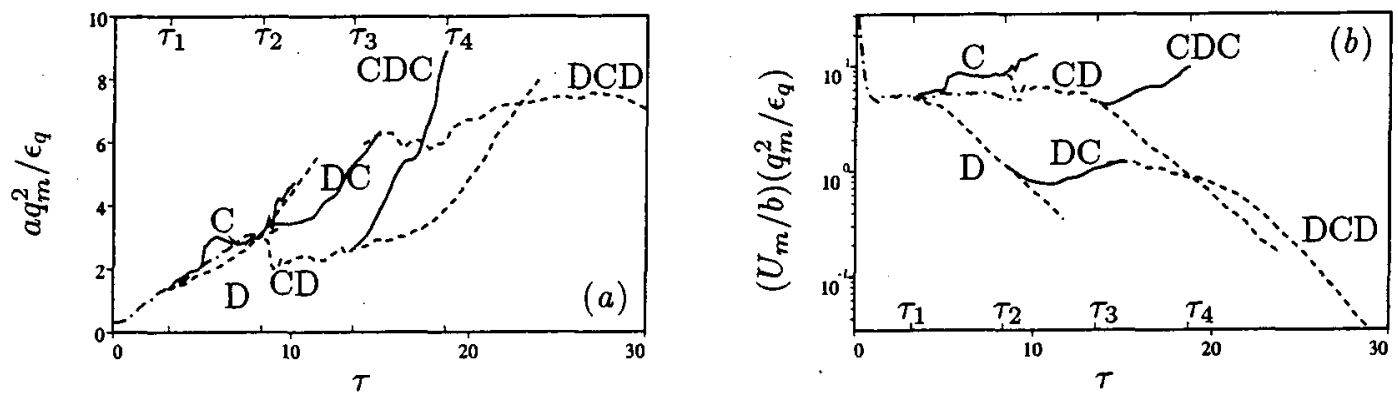

FIGURE 7. Turbulence time scale evolution at point of maximum (in $x_{2}$ ) $q^{2}$, normalized by $(a)$ the constant strain rate $a$ and $(b)$ the wake shear rate $U_{m} / b$. $-C$ " strain, ---- "D" strain, - - no strain. The same value of $a$ is used in $(a)$ for the unstrained wake.

values of $a q_{m}^{2} / \epsilon_{q}$ vary by less than an order of magnitude for the strained wake flows. In Rogers (2002) it was observed that this time scale increased for all orientations of applied strain, and that the rate of this increase was insensitive to the orientation of the strain. From figure $7(a)$ it can be seen that while $a q_{m}^{2} / \epsilon_{q}$ does generally increase, its value is dependent on the strain history. The growth in case CDC is delayed relative to that of case DC beyond $\tau_{3}$ and the late-time behaviours of cases CD and DCD are also quite different. In most of the flows, the time scale remains fairly constant for $a \Delta t \approx 1$ after one type of strain is replaced by another and this tends to spread out the curves in figure $7(a)$.

In many shear flows, the turbulence time scale comes into balance with the wake shear rate after the flow develops (e.g. the unstrained wake flow discussed here). The presence of strain in the flows examined here prevents this. The product of the wake shear rate $U_{m} / b$ and the turbulence time scale $q_{m}^{2} / \epsilon_{q}$ is shown in figure $7(b)$. The suppression of the wake with "D"-type straining leads to exponential decay of this product, although initiation of this decay is delayed for cases $C D$ and DCD, which have been previously strained. Although the wake shear becomes constant with " $\mathrm{C}$ "-type straining, the turbulence time scale continually increases (see figure $7(a)$ ), resulting in the sustained growth of $\left(U_{m} / b\right)\left(q_{m}^{2} / \epsilon_{q}\right)$. This growth occurs at a similar rate for all three "C"-type flows.

Ultimately, the turbulence Reynolds number $q^{4} /(\epsilon \nu)$ grows for all six strained flows. The "C"-type strain results in fairly rapid growth (although after a period of decay in case DC), whereas " $D$ "-type strain yields modest growth after an initial period of decay, the end result being Reynolds numbers that do not vary by more than a factor of about two throughout the evolution of each simulation. Thus, even though the wake is suppressed in the "D"-type cases, the production associated with the applied strain prevents the flow from relaminarizing.

\subsection{The Reynolds stresses}

According to the classical self-similar solution presented in section 2.2, the individual Reynolds stresses $\overline{u_{i}^{\prime} u_{j}^{\prime}}$ will all grow or decay in proportion to the turbulent kinetic energy examined above, resulting in constant Reynolds stress anisotropy. However, the turbulence in these strained wake flows is not evolving in accordance with the similarity solution (2.9). Even for the modest strain rates examined here, the turbulence production by the applied strain has a significant effect and no constant anisotropy state is achieved. Indeed, in some of the flows one of the Reynolds stress components may be decreasing while another is increasing. It is thus necessary to examine the full Reynolds stress tensor $\overline{u_{i}^{\prime} u_{j}^{\prime}}$, rather than just $q^{2}$, to understand the evolution of the turbulence.

The width of all the Reynolds stress profiles is well described by $b(t)$, the width derived 
from the mean wake velocity deficit profile. Thus scaling the cross-stream coordinate $x_{2}$ by $b(t)$ when plotting these profiles does a good job at collapsing the cross-stream extent of the various curves. On the other hand, scaling the Reynolds stresses by $U_{m}^{2}(t)$ does not collapse their cross-stream variation. In fact, as can be seen from figure 6 , scaling by $U_{m}^{2}(t)$ causes greater disparity between the Reynolds stress profiles at various times than is observed in the unscaled profiles.

In the case of "C"-type straining, the wake width increases (ultimately as $e^{a_{22} t}$ ) and the wake shear rate approaches a constant (see figure 5). Because of the continued presence of wake shear, there continues to be production in the $\overline{u_{1}^{\prime 2}}$ Reynolds stress component $\left(\mathcal{P}_{11}^{w}>0\right)$ and because this production is largest at the locations of maximum shear, the $\overline{u_{1}^{\prime 2}}$ profile tends to become at least slightly "double-peaked". The Reynolds stress component associated with the other homogeneous direction, $\overline{u_{3}^{\prime 2}}$ behaves similarly, although the growth is less pronounced. The behaviour of the cross-stream component $\overline{u_{2}^{\prime 2}}$ is more variable, with increasing levels in case $\mathrm{C}$, roughly constant levels in case CDC, and decreasing levels in case DC. The reduced levels of $\overline{u_{2}^{\prime 2}}$ in the latter two cases result in decreasing levels of the Reynolds shear stress $\overline{u_{1}^{\prime} u_{2}^{\prime}}$, in contrast to case $\mathrm{C}$, in which all the Reynolds stresses increase at roughly similar rates.

In contrast to the increasing wake widths of the "C"-type cases, the widths in the "D"-type cases are decreasing (or approaching a constant at late times). However, the turbulence is advected inward by the mean strain such that the width of the turbulent region is still well described by the width of the mean velocity profile, as noted above. In these " $\mathrm{D}$ "-type flows, the wake is suppressed, the wake shear is not maintained (figure 5), and the Reynolds shear stress is driven to zero. The production of turbulence ultimately results solely from the applied mean strain. The Reynolds stress profiles thus become single-peaked and the strongest growth, if any, is observed in the $\overline{u_{2}^{\prime 2}}$ component as a result of $\mathcal{P}_{22}^{s}$ being greater than zero. For this strain geometry, $\mathcal{P}_{11}^{s}$ is negative and this negative "production" results in the decay of $\overline{u_{1}^{\prime 2}}$ to low levels in all of the "D"-type cases.

The differing growth and decay rates of the various Reynolds stress components result in significant anisotropy of the Reynolds stress tensor in these strained wake flows. Switching the sign of the strain can reverse the growth or decay and leads to differing relative strengths of the various Reynolds stress components. This relative importance is easily visualized by examining plots of the Reynolds stress anisotropy tensor $b_{i j}$, where

$$
b_{i j}=\frac{\overline{u_{i}^{\prime} u_{j}^{\prime}}}{q^{2}}-\frac{\delta_{i j}}{3},
$$

$\delta_{i j}$ being the Kronecker delta. Scaled in this way, the values of $b_{i j}$ range between $-1 / 3$ and $2 / 3$ for the normal $(i=j)$ components and between $-1 / 2$ and $1 / 2$ for the shear $(i \neq j)$ components. The profile of the Reynolds shear stress $\overline{u_{1}^{\prime} u_{2}^{\prime}}$ (and therefore also $b_{12}$ ) is statistically anti-symmetric and its value at the centreline is near zero. Because of this, the anisotropies at the point of maximum $\overline{u_{1}^{\prime} u_{2}^{\prime}}$ are examined, rather than the centreline values.

The time evolution of the Reynolds stress anisotropy for all six simulations is depicted in figure 8. For case $\mathrm{C}$ (figure $8(a)$ ), the relative magnitudes of the various terms are similar to those of the unstrained wake (the initial values at $\tau_{1}$ ). Production by the applied strain tends increase the relative importance of $b_{11}\left(\mathcal{P}_{11}^{s}>0\right)$ and decrease that of $b_{22}\left(\mathcal{P}_{22}^{s}<0\right)$. Reversing the sign of the strain in case $\mathrm{D}$ leads to a re-ordering of the relative strengths of the stress components, with the strain production driving $b_{11}$ to low 

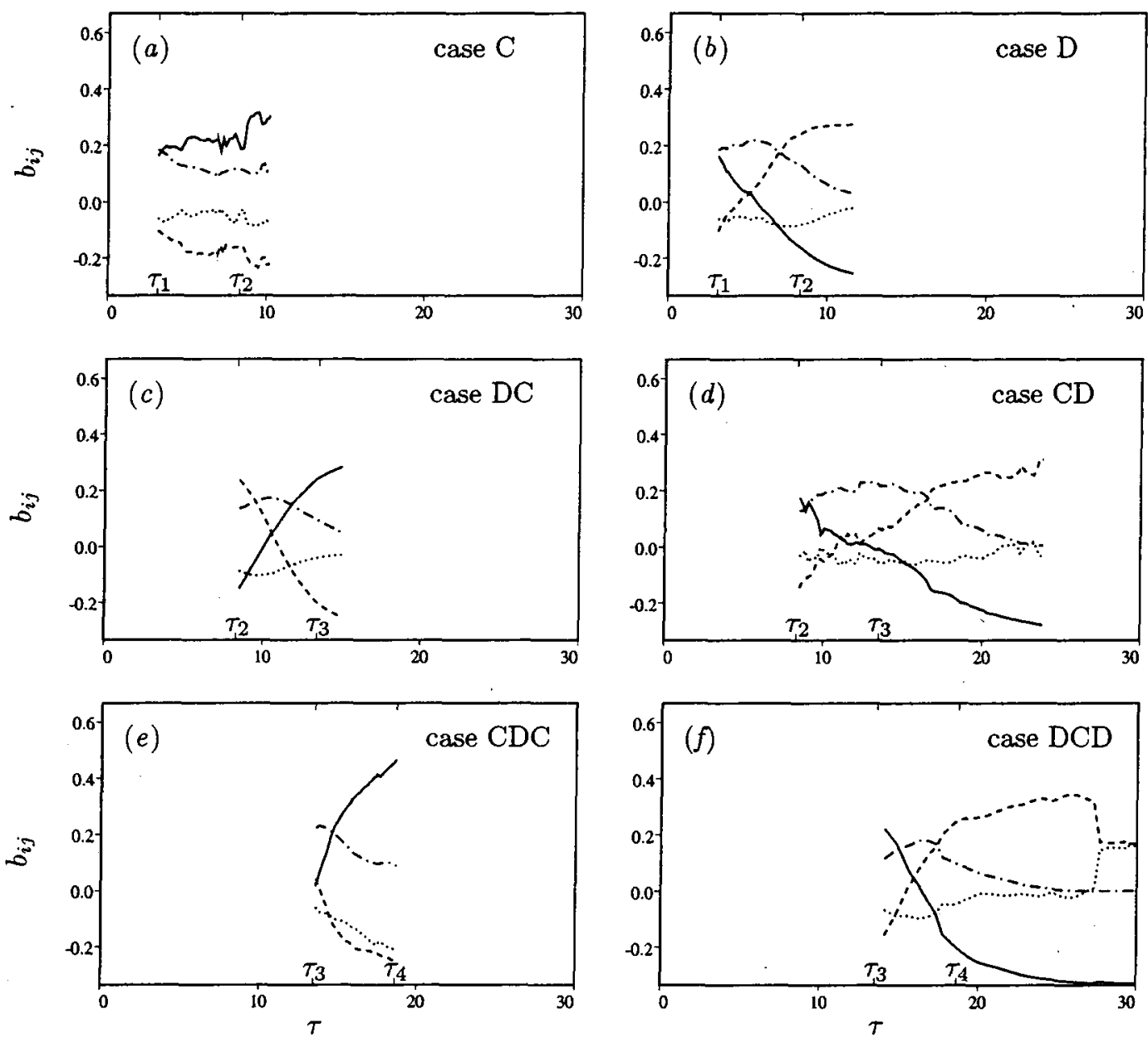

FIGURE 8. Reynolds stress anisotropy at point of maximum $\overline{u_{1}^{\prime} u_{2}^{\prime}}$ for $(a)$ case $C,(b)$ case $D,(c)$ case DC, $(d)$ case $\mathrm{CD},(e)$ case $\mathrm{CDC}$, and $(f)$ case DCD. $\longrightarrow b_{11},-\cdots b_{22}, \cdots \cdots b_{33}$, and $-b_{12}$.

levels, while increasing $b_{22}$ to values much larger than those of the other components. As with case $\mathrm{C}, b_{33}$ remains relatively unchanged, but the decay of the wake component drives $b_{12}$ to zero.

If the sign of the strain is reversed, and " $\mathrm{C}$ "-type strain is applied following the " $\mathrm{D}$ "type strain (figures $8(c)$ and $(e)$ ), then the ordering once again returns to $b_{11}>b_{33}>b_{22}$. Although the levels of $b_{12}$ decay, they are not driven to zero. When " $\mathrm{D}$ "-type strain is applied following "C"-type strain (figures $8(d)$ and $(f)$ ), the shear stress $b_{12}$ is driven to zero as the wake disappears and the energy in the streamwise fluctuations becomes negligible $\left(b_{11}\right.$ approaches $\left.-1 / 3\right)$. Because of production by the applied strain, $b_{22}$ increases to about $1 / 3$, while $b_{33}$ remains close to zero. (Note that the late time $\tau>27$ behaviour in figure $8(f)$ should be ignored because the point of maximum $\overline{u_{1}^{\prime} u_{2}^{\prime}}$ is no longer well-defined, having decayed to insignificant levels.)

It is thus apparent that, even at these modest strain rates, the anisotropy evolution is strongly affected by the production associated with the applied strain. The dominance of large-scale production mechanisms may explain the success of Rapid Distortion Theory (RDT) in describing such strained wake flows (Townsend 1980). It should also be noted 


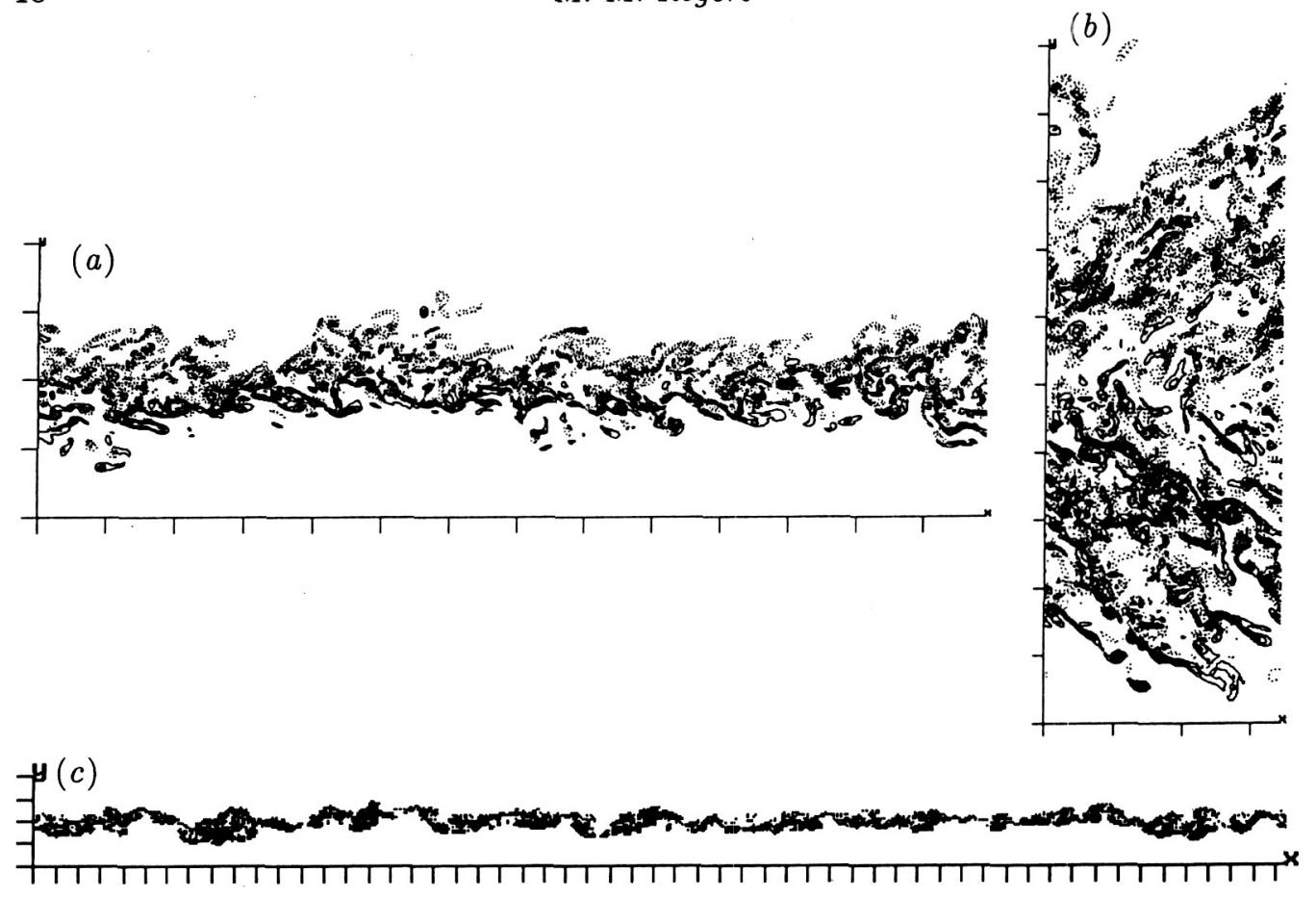

FIgURE 9. Contours of spanwise vorticity in the $x_{3}=0$ plane at $(a) \tau_{1}$ (initial condition), (b) $a\left(t-t_{1}\right)=1.40$ for case C, and $(c) a\left(t-t_{1}\right)=1.40$ for case D. Negative contours are dotted, positive contours are solid, and the contour increment is $3.0 U_{m}^{0} / b^{0}$ in $(a), 2.0 U_{m}^{0} / b^{0}$ in $(b)$, and $1.0 U_{m}^{0} / b^{0}$ in $(c)$. Tick marks are at $b^{0}$ intervals. Note that $(c)$ is not at the same scale as the other frames.

that the strong changes in component anisotropy levels brought about by the strain are inconsistent with the constant-anisotropy classical self-similar solution of section 2.2 . Apparently the turbulence "production" by the strain prevents this solution from being realized, at least for the strain rates considered here. In case $\mathrm{C}$ the variation of the anisotropy components is less than in the other flows, but the effects of strain production are still apparent in figure $8(\dot{a})$. It is possible that, after a long enough time, a constantanisotropy state might be achieved for the " $D$ "-type straining. However, this state $\left(b_{11}=\right.$ $-1 / 3, b_{12}=b_{33}=0$, and $b_{22}=1 / 3$ ) would be associated with a flow that no longer has a wake component.

\subsection{Flow visualization}

In this section contour plots of spanwise vorticity are used to visualize the structure of the strained wake flow fields. Instantaneous slices through the full computational domain at $x_{3}=0$ for each of the six strained wake simulations examined here are shown in fixed laboratory coordinates (not the moving computational coordinate system). The unstrained wake field used for the initial condition of the strained wake computations is shown in figure $9(a)$. Fields resulting from straining with "adverse" and "favourable" strain are shown in figures $9(b)$ and $(c)$, respectively. The images of these strained flows are similar to those shown in Rogers (2002), although the total strain here is larger 
$\left(a\left(t-t_{1}\right)=1.4\right.$, corresponding to a total strain of just over 4 , rather than $a\left(t-t_{1}\right)=1.2$, or a total strain of 3.3). Frames $(b)$ and $(c)$ thus represent the initial conditions for the successively strained cases CD and DC, respectively. As discussed in Rogers (2002), "type-C" straining ultimately results in a thick wake with an exponentially increasing width, which follows the distortion associated with the mean strain. The streamwise domain size becomes limited and at late times the simulation may no longer provide an adequate approximation to the infinite-domain problem. However, there is not much large-scale organization present during the flow's evolution and this domain size limitation may not be as severe as suggested by the ratio of the flow width to the streamwise domain extent. Many small-scale motions contribute to an eddy viscosity type of mixing and the associated passive scalars contours are roughly horizontal, without large incursions of relatively unmixed fluid from opposite sides of the wake. For case $D$, the strain results in an ever-increasing streamwise domain extent and a corresponding increase in the statistical sample of eddies. Despite the compressive straining in the cross-stream $\left(x_{2}\right)$ direction, the wake width does not continually diminish, but reaches a roughly constant thickness. The wake itself maintains some large-scale organization, with small-scale vortical motions "clumping" into larger organized motions and unmixed free-stream fluid from both sides of the wake penetrating across the wake centreline.

The response of the turbulence to successive straining is depicted by the spanwise vorticity contours shown in figures 10 and 11. Starting from the field in figure $9(b)$, case CD is "favourably" strained until the original streamwise domain extent is recovered, corresponding to an overall applied total strain of one (figure 10(b)). At this point, the flow is once again strained "adversely" (case CDC) until a time $\tau_{4}$ at which $a\left(t-t_{3}\right)=1.4$ once again (figure $10(d)$ ). The turbulence is dominated by small-scale motions with little large-scale coherence, and the vortical eddies seem to passively follow the mean distortion brought about by the applied strain. Under the action of the "favourable" strain the vortical eddies become elongated in the streamwise direction. In response to the "adverse" strain the eddies become more vertically oriented.

Again, the lack of large-scale coherence results in an eddy-viscosity type of mixing, with relatively horizontal passive scalar isosurfaces. Fluid from one side of the wake does not penetrate far across the wake without becoming mixed. Once the "adverse" straining is begun the passive scalar contours appear to "buckle" and collapse on each other, temporarily permitting deeper incursions of fluid across the wake (at the time of figure $10(c)$ ) before finally ending up in a pattern at $\tau_{4}$ (figure $10(d)$ ) that is similar to that of case $\mathrm{C}$ at $\tau_{2}$ (figure $9(b)$ ). This "buckling" behaviour is more pronounced in case DC described below.

The total strain of case CD at $\tau_{3}$ corresponds to that of the initial flow field at $\tau_{1}$. Contour plots of the spanwise vorticity for these two fields are similar (compare figure $10(b)$ with figure $9(a)$ ), although the wake width of case $C D$ at $\tau_{3}$ is slightly larger, as also noted from figure 3. Similarly, the total strain of case CDC at $\tau_{4}$ is the same as that of the case $\mathrm{C}$ at $\tau_{2}$. Again, both the vorticity and passive scalar fields are similar (compare figure $10(d)$ with figure $9(b))$ and the flow width at the later time is slightly greater. 

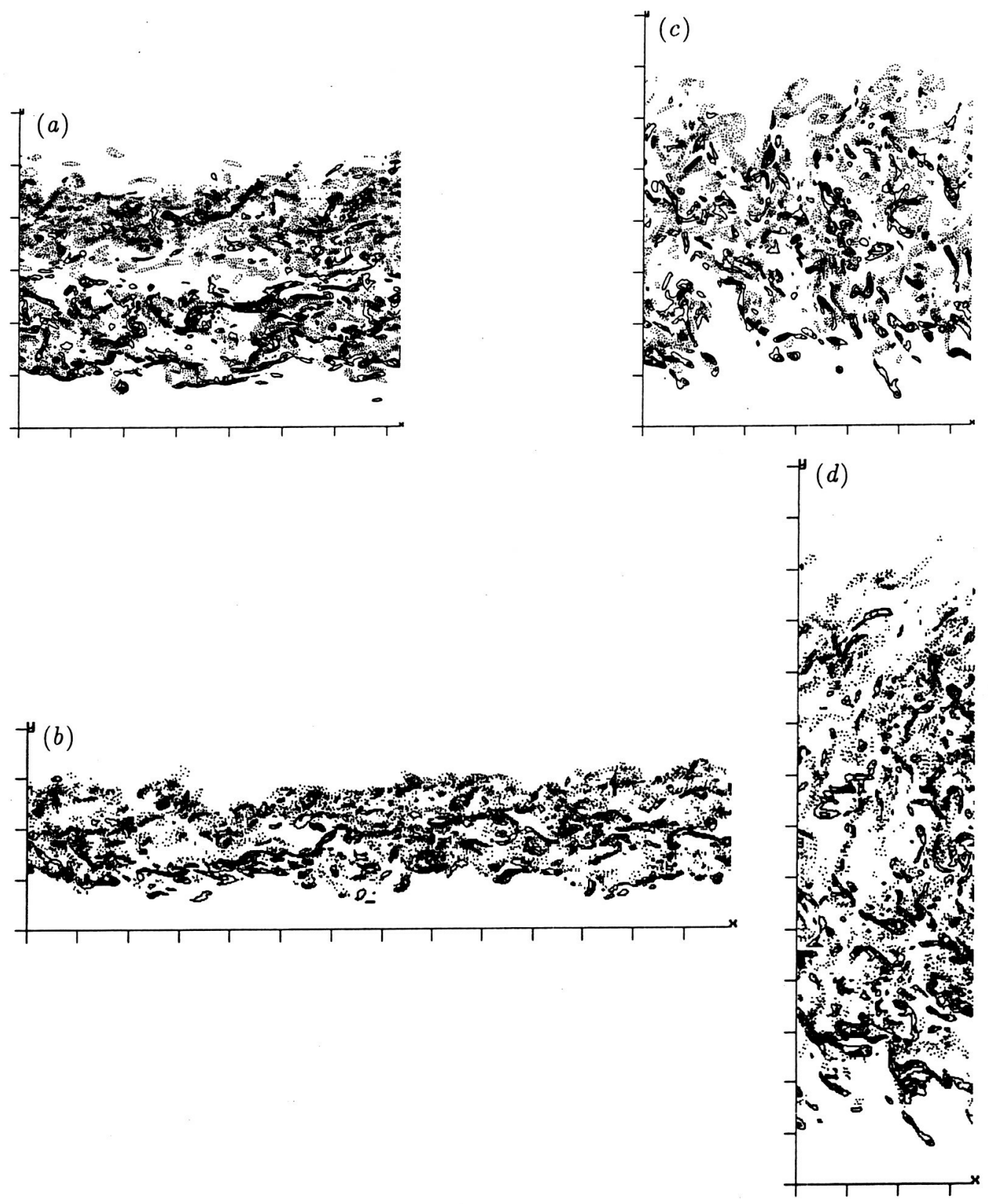

FIGURE 10. Contours of spanwise vorticity in the $x_{3}=0$ plane at $(a) a\left(t-t_{2}\right)=0.75$ for case CD, (b) $a\left(t-t_{2}\right)=1.40$ for case CD, (c) $a\left(t-t_{3}\right)=0.78$ for case CDC, and (d) $a\left(t-t_{3}\right)=1.40$ for case CDC. Negative contours are dotted, positive contours are solid, and the contour increment is $2.0 U_{m}^{0} / b^{0}$. Tick marks are at $b^{0}$ intervals. 

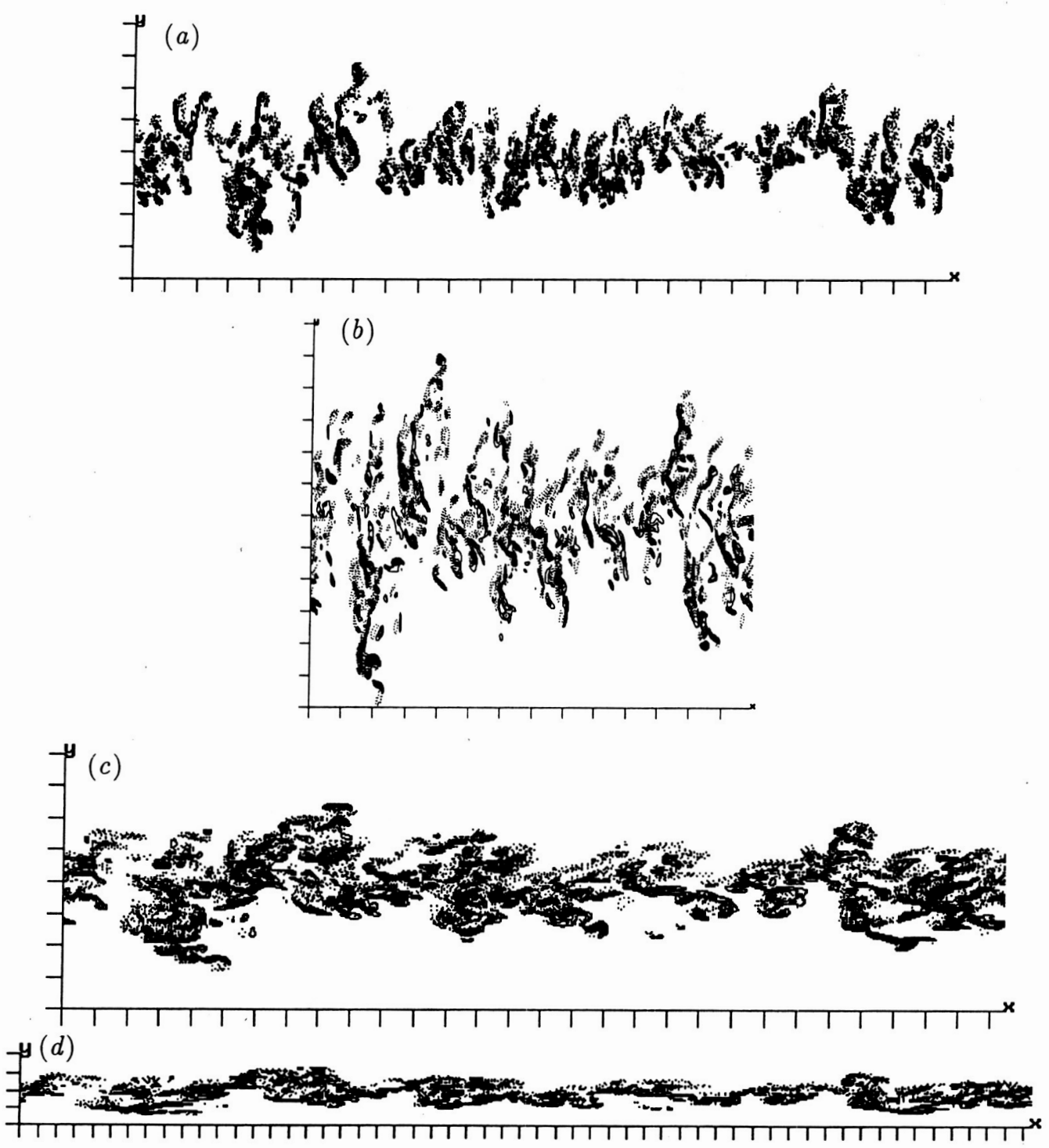

FIGURE 11. Contours of spanwise vorticity in the $x_{3}=0$ plane at $(a) a\left(t-t_{2}\right)=0.78$ for case DC, (b) $a\left(t-t_{2}\right)=1.40$ for case DC, (c) $a\left(t-t_{3}\right)=0.75$ for case DCD, and $(d) a\left(t-t_{3}\right)=1.40$ for case DCD. Negative contours are dotted, positive contours are solid, and the contour increment is $0.5 U_{m}^{0} / b^{0}$ for case DC and $0.25 U_{m}^{0} / b^{0}$ for case DCD. Tick marks are at $b^{0}$ intervals. Note that $(d)$ is not at the same scale as the other frames.

The evolution of the spanwise vorticity in cases DC and DCD is shown in figure 11. The initial state for this pair of runs is shown in figure 9(c). "Adverse" straining compresses the flow in the streamwise direction and the row of "clumped" vortices appears to "buckle" into a series of vertically oriented eddies. Contours of the passive scalar quantity (not shown) wind sinuously up and down across the wake, with significant pockets of unmixed fluid from one side of the wake penetrating well across the centreline. After the "adverse" strain has been applied for $a\left(t-t_{2}\right)=1.40$, the total strain again reaches one (figure $11(b)$ ), with the streamwise domain extent being equal to its size in the ini- 


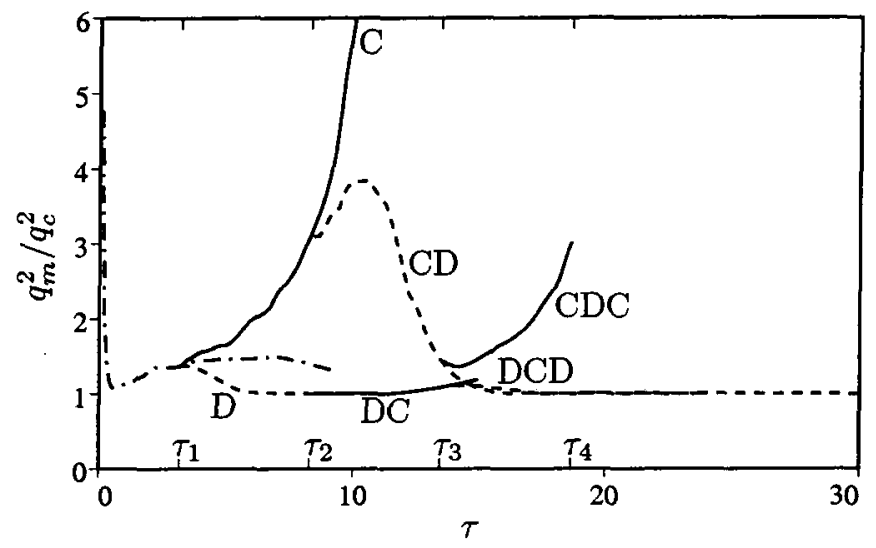

FIGURE 12. Evolution of the ratio between the maximum (in $x_{2}$ ) of the turbulent kinetic energy and its centreline value. — " $C$ " strain, --- " $\mathrm{D}$ " strain, - - - no strain.

tial unstrained wake (figure $9(a)$ ). The turbulence at this point, however, is markedly different from that of the unstrained wake at $\tau_{1}$. The wake width is 4.3 times that at $\tau_{1}$ and the vortical motions are vertically oriented, with deep incursions of unmixed fluid across the wake as noted above. Additionally, the vorticity has decreased in magnitude, with the contour levels in figure $11(b)$ being only a sixth of those used in figure $9(a)$. At $\tau_{3}$, "favourable" straining is once again applied and the eddies again become elongated in the streamwise direction, with little unmixed fluid penetrating across the wake centreline. At $\tau_{4}$, the total strain for case DCD (figure $11(d)$ ) is the same as that for case $\mathrm{D}$ at $\tau_{2}$ (figure $9(c)$ ). The structure of these two "favourably" strained flowfields is also similar, despite the differences in their prior history. The eddies are elongated in the streamwise direction and there is some large-scale "clumping". At $\tau_{4}$, the wake is $60 \%$ wider than at $\tau_{2}$, but this is a relatively small difference given that the wake grew in width by a factor of five and a half by $\tau_{3}$ (see figure 3). Careful examination of the contour plots does reveal some differences, however. The larger, slower (see figure $7(a)$ ) large-scale eddies in case DCD tend to be more smeared out in the streamwise direction, whereas the smaller, faster eddies in case D are not as passively advected by the strain, maintaining less elongated shapes more typical of those found in unstrained wakes.

As is readily seen from the above flow visualizations, the primary effect of the applied strain is a "box distortion" of the vortical eddies. They become elongated in directions of expansive strain and compact in directions of compressive strain. Thus, for the strain rates considered here, the turbulence reacts fairly slowly to the applied strain and is, to first-order, passively advected by it. As the simulations proceed to later and later times this effect becomes even more pronounced because the turbulence time scale increases, as noted above. Even for the modest strain rates considered here, the turbulence is unable to stay in equilibrium with the rapidly changing mean flow. This is also true for orientations of applied strain other than those considered here (Rogers 2002).

In Rogers (2002) it was noted that in case $\mathrm{C}$ the strain resulted in a $q^{2}$-profile that was double-peaked, with turbulent kinetic energy levels at the centreline, $q_{c}^{2}$, decreasing despite growth of the peak turbulent kinetic energy level $q_{m}^{2}$. This leads to a relatively less active zone near the wake centreline for this case (as can be seen in figure $9(b))$. The ratio $q_{m}^{2} / q_{c}^{2}$, a good indicator of this effect, is shown in figure 12 for all six strained flows examined here as well as for the unstrained wake. While this ratio is fairly constant for the unstrained wake (as expected for self-similar $q^{2}$ profiles), it increases for all cases subjected to "C"-type strain. In contrast, the ratio $q_{m}^{2} / q_{c}^{2}$ for cases with "D"-type strain 

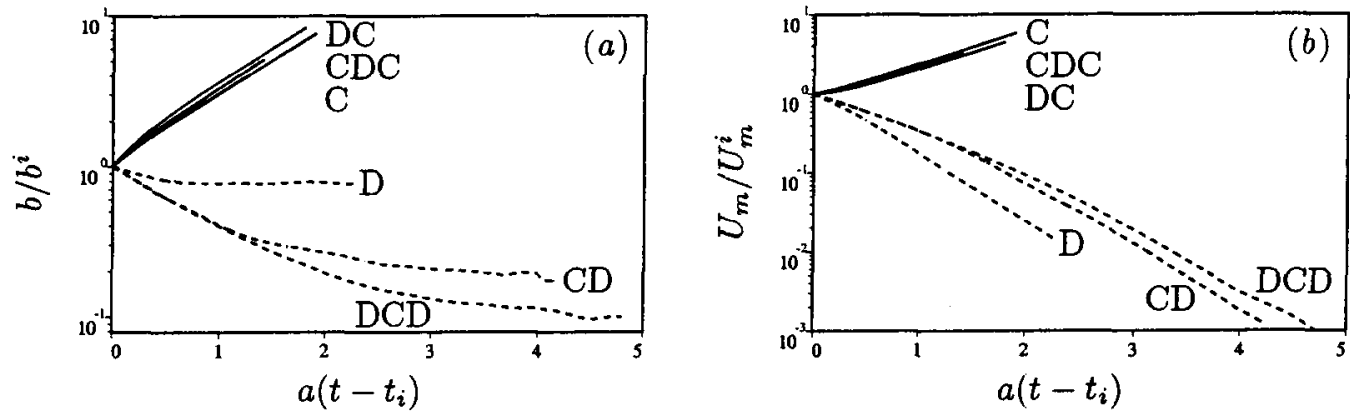

FIGURE 13. Shifted and scaled wake $(a)$ width and $(b)$ peak velocity deficit evolution. "C" strain, -..- "D" strain.

is rapidly driven to one, indicating that the peak of the profile moves to the centreline in these cases. However, even if the peak has moved to the centreline, application of "C"-type strain can still recreate a double-peaked profile (as in case DC, in which the value of $q_{m}^{2} / q_{c}^{2}$ is back up to 1.19 by the end of the simulation).

\section{Conclusions}

Plane wakes subjected to the successive application of two types of plane strain have been simulated using direct numerical simulation. One type of plane strain consists of compressive strain in the streamwise direction and expansive strain in the cross-stream direction, corresponding to a spatially evolving wake experiencing an adverse pressure gradient; the other type is opposite in sign, analogous to a spatially evolving wake experiencing a favourable pressure gradient. The magnitude of the strain rate $a$ is the same for both types.

Simulations are begun from five different initial conditions. Two are begun from the unstrained plane wake at time $t_{1}$. Two are begun from cases that have experienced a total strain $e^{a \Delta t}$ of about 4 (or $\frac{1}{4}$ ) at time $t_{2}$ and two are begun from cases that have returned to a total strain of one at time $t_{3}$. Comparing the responses of these different flows to the two types of strain considered here will thus address the sensitivity of the evolution to different turbulent initial conditions as well as the importance of the strain-rate history in determining the flow evolution.

The characteristic shape of the mean wake velocity profile $(f(\eta)$ in equation $(2.9))$ was found to be nearly universal, both throughout the evolution of each individual flow and between flows. As a result of this, the Reynolds number $R e_{m}=U_{m} b / \nu$, where $U_{m}$ is the peak wake velocity deficit and $b$ is the wake half-width, changes exponentially as $e^{2 a_{22} t}$. Additionally, because of this, $R e_{m}$ is determined entirely by the total strain experienced by the flow (as shown in figure 1); the strain-rate history is irrelevant. The value of $R e_{m}$ at $t_{3}$ is the same for both cases CD and DC because the total strain has returned to one in both these flows at that time. The subsequent evolution of $R e_{m}$ in both these cases is identical to that of cases DCD and CDC, respectively, because the total strain histories are the same. The order in which the strains are applied does not matter because the effects on $R e_{m}$ are reversible.

The strain history does have an impact on the evolutions of the wake width $b(t)$ and the velocity deficit $U_{m}(t)$, however. The evolutions of these quantities for the various cases, shown in figures 3 and 4 , have been replotted slightly differently in figures 13a and $13 \mathrm{~b}$ to facilitate comparison of the responses to each type of applied strain. In these figures the time origins have been shifted by $t_{i}$, the time at which the current strain was 
first applied, so that each curve begins at $t-t_{i}=0$. Also, the initial amplitudes $b^{i}$ and $U_{m}^{i}$ at the time $t_{i}$ have been used to scale $b$ and $U_{m}$, respectively, so that each curve begins at one.

The response to " $\mathrm{C}$ "-type (adverse) straining shows little variation among the three cases simulated. Despite the different initial turbulence in these cases (unstrained wake turbulence, previously strained wake turbulence stretched in the streamwise direction to a total strain of four, and previously successively strained turbulence at a total strain of one), the growth of $b$ and $U_{m}$ is similar in all three flows. After a transient period of $a \Delta t \approx 0.5$, both of these quantities evolve exponentially with $n_{b}=1$ and $n_{U}=1$. During this transient period, the values of $n_{b}$ and $n_{U}$ are, respectively, somewhat larger and smaller than these asymptotic values. The good agreement between these three cases suggests that the wake width and deficit can be well-predicted during the application of adverse straining, with both these measures fairly quickly growing at the rate the flow is being stretched by the cross-stream expansive strain.

The response to " $\mathrm{D}$ "-type (favourable) straining, on the other hand, is more sensitive to the strain history of the flow. Although the exponential growth rates evolve towards $n_{b}=$ 0 and $n_{U}=-2$ in all three cases, the time required to achieve this asymptotic behaviour varies widely (note $n_{b}+n_{U}=-2$ as a result of equation (2.5) and the universality of $f(\eta))$. In case $D$ this asymptotic growth rate is achieved after $a \Delta t \approx 0.5$ and $b$ never decays more rapidly than $n_{b}=-0.5$. For the previously strained cases CD and DCD, the wake width does not become approximately constant until $a \Delta t \approx 4$, with an initial period of about $a \Delta t \approx 1$ during which the width decays rapidly with $n_{b}=-1$. Thus if the turbulence has been previously stretched in the cross-stream direction it can be quickly recompressed if the sign of the strain changes. If the turbulence is in equilibrium with the mean flow, as in the self-similar unstrained wake, then it exhibits a greater resistance to compression in the cross-stream direction.

In all cases the ultimate values of $n_{b}$ and $n_{U}$ are the same as those reported for cases C and D in Rogers (2002). The classical self-similar solution for strained wakes thus does not describe the ultimate flow evolution, whereas alternative equilibrium similarity solutions can. It is interesting to note, however, that during the transitional period of the favourably strained cases CD and DCD when $n_{b}=n_{U}=-1$, the evolutions are in agreement with the predictions of the classical similarity solution (they are also in agreement with the predictions of the equilibrium similarity solution (2.11) if $E_{2}=0$ ). During this time, it appears that the turbulence is being passively squeezed after having been rapidly stretched. It is not until the turbulence is able to resist the cross-stream compression that the wake width predictions of the classical similarity solution become inaccurate.

The varied response to the cross-stream compression associated with favourable straining results in wake width and velocity deficit evolutions that are sensitive to the strain history. The order in which various strains are applied can change the end state and $b$ and $U_{m}$ are not completely determined by the total strain. As noted in the discussion of figures 3 and 4 , the values of $b$ and $U_{m}$ at $\tau_{3}$ (total strain of one) differ by a factor of about three. Because of the different responses to compressive cross-stream straining, the trajectories in these figures do not close on each other at $\tau_{3}$ as they did for $R e_{m}$ and the effects of straining on $b$ and $U_{m}$ are, in general, not reversible. On the other hand, owing to the fairly extensive periods of $n_{b}=n_{U}=-1$ behaviour in cases CD and DCD, the strain effects are nearly reversible in some cases. For instance, the values of $b$ and $U_{m}$ for case CD at $\tau_{3}$ are only slightly different from their initial values at $\tau_{1}$, the values for case DCD at $\tau_{4}$ are only slightly different from those of case $\mathrm{D}$ at $\tau_{2}$, and the values for case CDC at $\tau_{4}$ are only slightly different from those of case $\mathrm{C}$ at $\tau_{2}$. Additionally, these 

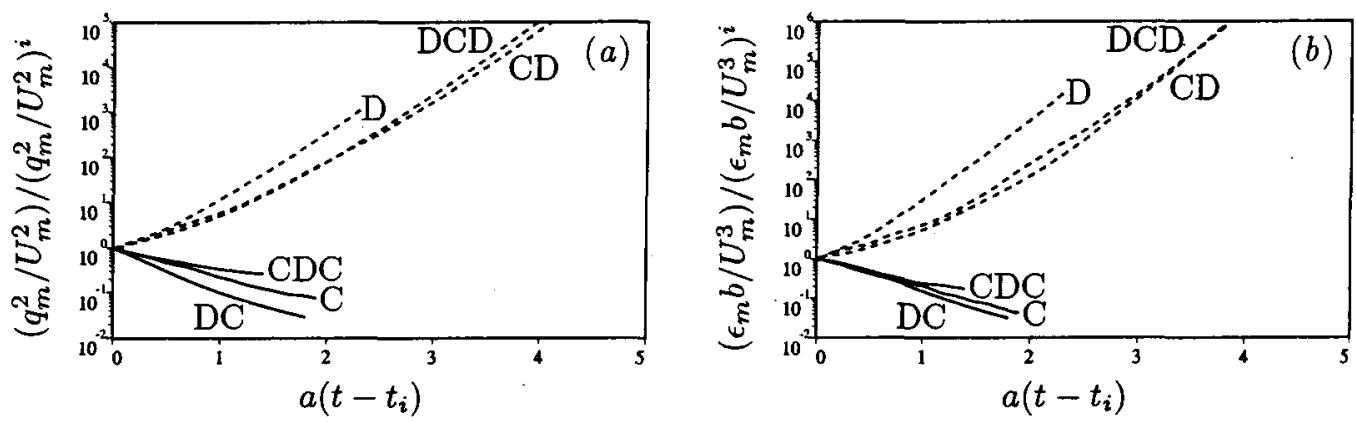

FIGURE 14. Shifted and scaled peak turbulence (a) kinetic energy $q_{m}^{2}$ and $(b)$ dissipation $\epsilon_{m}$, normalized by $b$ and $U_{m}$. evolution. - "C" strain, ---- "D" strain.

differences are in line with what would be expected for the spreading of an unstrained wake over the same time interval (increases of about $50 \%$ to $60 \%$ for $b$ and decreases of about $35 \%$ for $U_{m}$ ). The biggest irreversibility results when favourable straining is applied to previously unstrained turbulence, followed by adverse straining. In this case the wake fairly quickly resists the initial cross-stream compression (note the different trajectories for case $D$ in figures $13 \mathrm{a}$ and $13 \mathrm{~b}$ ) but not the ensuing cross-stream expansion associated with the subsequent adverse straining. The end result for case DC is a wake width at $\tau_{3}$ that is over four times that at $\tau_{1}$ and a velocity deficit that is reduced by over $75 \%$ between the same two times.

The effect of straining on the turbulent kinetic energy is dependent on the strain history and is, in general, not reversible. As can be seen in figure $6 \mathrm{~b}$, the magnitude of $q_{m}^{2}$ is different by nearly an order of magnitude between cases CD and DC at $\tau_{3}$. The value of $q_{m}^{2}$ at $\tau_{3}$ is close to the initial value at $\tau_{1}$ (reduced by less than $3 \%$ ), but none of the other pairs of strains have a reversible effect on this quantity. The response to both " $\mathrm{C}$ " and " $\mathrm{D}$ "-type straining is not universal, with the application of "C"-type straining to case $\mathrm{D}$ at $\tau_{2}$ actually resulting in an initially decreasing turbulent kinetic energy level, in contrast to the other two " $\mathrm{C}$ " cases, which exhibit increasing energy levels from the moment the strain is first applied.

Surprisingly, scaling $q_{m}^{2}$ by $U_{m}^{2}$ greatly reduces the dependence on the strain history (figure 6a). The values of $q_{m}^{2} / U_{m}^{2}$ at $\tau_{3}$ for cases CD and DC differ by only $15 \%$. The $q_{m}^{2} / U_{m}^{2}$ curves have been replotted in figure $14(a)$, shifted so that each begins at the origin with an initial value of one. From this it is clear that the evolutions are not identical for each type of strain, although they are qualitatively similar. As with the velocity deficit $U_{m}$ (figure $13 \mathrm{~b}$ ), the evolution for case $\mathrm{D}$ is different from the other two " $\mathrm{D}$ "-type cases. However, the more rapid increase in $q_{m}^{2} / U_{m}^{2}$ for case $\mathrm{D}$ is offset by the more rapid decrease for case DC, yielding a result at $\tau_{3}$ that is very similar to that achieved by the slightly slower decrease for case $\mathrm{C}$ followed by the slightly slower increase for case CD. Despite the somewhat different trajectories in figure $14 \mathrm{a}$, the value of $q_{m}^{2} / U_{m}^{2}$ is reasonably well determined by the total strain experienced by the wake. The effects of strain on this statistic appear largely reversible.

The magnitude of the dissipation rate of turbulent kinetic energy $\epsilon_{m}$ is also sensitive to the strain history. Like $q_{m}^{2}$, the values $\epsilon_{m}$ at $\tau_{3}$ differ by an order of magnitude, the value for case $\mathrm{DC}$ decays much more than that in the other " $\mathrm{C}$ "-type cases, and the value for case DCD ultimately increases, unlike in the other " $D$ "-type cases. Again, however, scaling by $b$ and $U_{m}$ results in evolutions that are more universal, although the evolution in case $D$ is again different from that of the other " $D$ "-type cases (figure 14b). Because of 
this, following case D with "C"-type straining in case DC yields a higher level of $\epsilon_{m} b / U_{m}^{3}$ at $\tau_{3}$ than following case $\mathrm{C}$ with " $\mathrm{D}$ "-type straining in case $\mathrm{CD}$.

Classical self-similar theory predicts that the ratios $q_{m}^{2} / U_{m}^{2}$ and $\epsilon_{m} b / U_{m}^{3}$ should be constant. This is not observed in the simulations. In all the "favourably" strained cases these ratios grow rapidly, whereas for the "adversely" strained cases they decay rapidly. The turbulence is unable to keep pace with the exponentially varying mean flow quantities $U_{m}$ and $b$.

The turbulence time scale $q_{m}^{2} / \epsilon_{q}$ changes fairly slowly in comparison with the other turbulent statistics and its evolution is apparently not strongly coupled to the mean strain. Different applied strains can result in similar time scale growth, although this growth often ceases for a period of about $a \Delta t=1$ after the sign of the applied strain is changed. Variability in this delay leads to dependence on the strain history. The weak connection between the applied strain and the resulting time scale evolution provides further evidence that the turbulence is not strongly coupled to the rapidly changing mean flow.

Flow visualization also indicates that to first order the turbulent eddies are largely advected by the mean strain rather than rapidly evolving in response to it. When streamwise compression is applied following streamwise expansion the vortical flow "buckles", resulting in deep incursions of freestream fluid into the layer (figure 11). Reversing the sign of the strain again at this point leads to a more extended period of reduction in wake width (with $n_{b}=-1$ ) than results when such favourable straining is applied to a previously unstrained wake, as discussed above. It is not until coherent larger scale motions reform that the wake resists further compression $\left(n_{b} \approx 0\right)$.

Although Townsend (1954) felt that his strained homogeneous turbulence attained a structural equilibrium with constant Reynolds stress anisotropies for total strains somewhat greater than two, later experiments by Tucker \& Reynolds (1968) and Marechal (1972) demonstrated that these anisotropies continue to increase with further increases in total strain. For the total strains examined here (see Table 1) there is no evidence of structural equilibrium in these strained shear flows. Reversing the sign of the applied strain results in rapid changes in anisotropy and the relative importance of the different Reynolds stress components. In their experiments on successively strained homogeneous turbulence, Gence \& Mathieu (1979) found that the observed anisotropy returned to its original minimal level when the total strain returned to one. This reversibility and dependence on only the total strain were consistent with RDT predictions. The evolution of the anisotropy in the strained wakes examined here is more complex, being a function of the strain history as well as the total strain. For instance, the anisotropies $b_{11}$ and $b_{22}$ of cases CD and DC at $\tau_{3}$ are not even of the same sign, despite the total strain being one in both cases. The production of Reynolds stress by the mean wake shear thus complicates the anisotropy evolution compared to that in unsheared turbulence.

The computer time required to generate these numerical simulations was provided by the NAS facility at the NASA Ames Research Center. 


\section{REFERENCES}

Beharelle, S., Nayeri, C. N., Delville, J., Bonnet, J.P. \& Fiedler, H. E. 1996 Influence of the transverse shear on the development of wake flows. In Advances in Turbulence VI (ed. S. Gavrilakis, L. Machiels, P. A. Monkewitz), pp. 511-512. Kluwer Academic Publishers.

Atsavapranee, P. \& Gharib, M. 1997 Structures in stratified plane mixing layers and the effects of cross-shear. J. Fluid Mech. 342, 53-86.

Corral, R. \& JiMENEZ, J. 1995 Fourier/Chebyshev methods for the incompressible NavierStokes equations in infinite domains. J. Comput. Physics 121, 261-270.

ELLIOTT, C. J. \& TOWNSEND, A. A. 1981 The development of a turbulent wake in a distorting duct. J. Fluid Mech. 113, 433-467.

Gence, J. N. \& MATHIEU, J. 1979 On the application of successive plane strains to gridgenerated turbulence. J. Fluid Mech. 93, 501-513.

Hill, P. G., Schaub, U. W. \& Senoo, Y. 1963 Turbulent wakes in pressure gradients. Trans. ASME E: J. of Appl. Mech. 30, 518-524.

Hoffenberg, R, Sullivan, J. P. \& Schneider, S. P. 1995 Wake measurements in a strong adverse pressure gradient. AIAA Paper 95-1912.

KefFer, J. F. 1965 The uniform distortion of a turbulent wake. J. Fluid Mech. 22, 135-159.

KEFFER, J. F. 1967 A note on the expansion of turbulent wakes. J. Fluid Mech. 28, 183-193.

Keffer, J. F., Kawali, J. G., Hunt, J. C. R. \& MaxeY, M. R. 1978 The uniform distortion of thermal and velocity mixing layers. J. Fluid Mech. 86, 465-490.

MARECHAL, J. 1972 Etude experimentale de la deformation plane d'une turbulence homogene. J. Mec. 11, 263-294.

Moser, R. D., Rogers, M. M. \& EwING, D. W. 1998 Self-similarity of time-evolving plane wakes. J. Fluid Mech. 367, 255-289.

Nayeri, C., Beharelde, S., Delville, J., Bonnet, J.P. \& Fiedler, H. E. 1996 Experimental investigation of the axisymmetric shear layer between coaxial jets with swirl. In Proc. ETC$V I$, Kluwer Academic Publishers.

REYNolds, A. J. 1962 Observations on distorted turbulent wakes. J. Fluid Mech. 13, 333-355.

ROGALLO, R. S. 1977 An ILLIAC program for the numerical simulation of homogeneous incompressible turbulence. NASA TM 73203.

Rogallo, R. S. 1981 Numerical experiments in homogeneous turbulence. NASA TM 81315.

Rogers, M. M. 2002 The evolution of strained turbulent plane wakes. J. Fluid Mech. 463, $53-120$.

RUMSEY, C. L. \& GATSKI, T. B. 2000 Recent turbulence model advances applied to multielement airfoil computations. AIAA Paper 2000-4323.

Townsend, A. A. 1954 The uniform distortion of homogeneous turbulence. Quart. J. Mech. Appl. Math. 7, 104-127.

TownsEnd, A. A. 1980 The response of sheared turbulence to additional distortion. J. Fluid Mech. 81, 171-191.

TUCKER, H. J. \& REYNOLDS, A. J. 1968 The distortion of turbulence by irrotational plane strain. J. Fluid Mech. 32, 657-673. 